\title{
Statistics of Co-Channel Interference in a Field of Poisson and Poisson-Poisson Clustered Interferers
}

\author{
Kapil Gulati, Student Member, IEEE, Brian L. Evans, Fellow, IEEE, Jeffrey G. Andrews, Senior Member, IEEE, \\ and Keith R. Tinsley, Senior Member, IEEE
}

\begin{abstract}
With increasing spatial reuse of radio spectrum, cochannel interference is becoming a dominant noise source and may severely degrade the communication performance of wireless transceivers. In this paper, we consider the problem of statisticalphysical modeling of co-channel interference from an annular field of Poisson or Poisson-Poisson cluster distributed interferers. Poisson and Poisson-Poisson cluster processes are commonly used to model interferer distributions in large wireless networks without and with interferer clustering, respectively. Further, by considering the interferers distributed over a parametric annular region, we derive interference statistics for finite- and infinitearea interference region with and without a guard zone around the receiver. Statistical modeling of interference is a useful tool to analyze outage probabilities in wireless networks and design interference-aware transceivers. Our contributions include (1) developing a unified framework for deriving interference models for various wireless network environments, (2) demonstrating the applicability of the symmetric alpha stable and Gaussian mixture (with Middleton Class A as a particular form) distributions in modeling co-channel interference, and (3) deriving analytical conditions on the system model parameters for which these distributions accurately model the statistical properties of the interference. Applications include co-channel interference modeling for various wireless networks, including wireless ad hoc, cellular, local area, and femtocell networks.
\end{abstract}

Index Terms-Co-channel interference, Poisson processes, impulsive noise, probability, stochastic approximation.

\section{INTRODUCTION}

Current and future wireless communication systems require higher spectral usage due to increasing demand in user data rates. One of the principal techniques for efficient spectral usage is to implement a dense spatial reuse of the available radio spectrum. This causes severe co-channel interference, which limits the communication system performance. Knowledge of interference statistics is integral to analyzing performance of wireless networks, including outage probability and throughput, and can also be used to design transceivers with improved communication performance [1]-[6]. We have released a freely distributed software toolbox for statistical modeling and mitigation of radio frequency interference [7].

Copyright (c) 2010 IEEE. Personal use of this material is permitted. However, permission to use this material for any other purposes must be obtained from the IEEE by sending a request to pubs-permissions@ieee.org.

K. Gulati, B. L. Evans, and J. G. Andrews are with the Department of Electrical and Computer Engineering, The University of Texas at Austin, TX, 78712 USA e-mail: \{gulati, bevans, jandrews\} @ece.utexas.edu.

K. R. Tinsley is with Intel Corporation, Santa Clara, CA 95054 USA e-mail: keith.r.tinsley@intel.com.

This research was supported by Intel Corporation.

Manuscript submitted November 29, 2009; revised April 3, 2010; accepted August 12, 2010.

\section{A. Motivation and Prior Work}

Co-channel interference statistics in wireless networks are affected by the following key factors: (i) the spatial distribution of interferers, (ii) the spatial region over which the interferers are distributed, and (iii) propagation characteristics including the power pathloss exponent and fading. Regarding (i), the distribution of active interferers in large random wireless networks is generally assumed to be a homogeneous spatial Poisson point process [6], [8]-[11]. While this assumption may be valid for certain wireless networks (e.g. wireless sensor and ad hoc networks), it may be common for interfering users to cluster in space due to geographical factors (e.g. gathering places or femtocell networks [12], [13]), or medium access control (MAC) layer protocols [6], [14]. Regarding (ii), the spatial region containing the interferers is commonly assumed to be an infinite plane [8]-[11]. Many wireless networks, however, employ contention-based MAC protocols (e.g. carrier sense multiple access and multiple access with collision avoidance) or other local coordination techniques to limit the interference, thereby creating a guard zone around the receiver (e.g. in wireless ad hoc networks [15] and in dense Wi-Fi networks [6], [16]). Guard zones around the receiver can also occur due to scheduling-based MAC protocols, such as in cellular networks in which the users in the same cell site are orthogonal to each other and all interfering users are outside the cell site in which the receiver is located. Further, receivers in many wireless networks may experience interference from finite-area regions (e.g. interference from a cell cite in cellular networks with reuse factor greater than one) [17]. This motivates characterizing the interference statistics in Poisson and Poisson-Poisson clustered interferers distributed over a parametric annular region. For each of the interferer distributions, the finite- and infinite- area with and without a guard zone around the receiver can then be studied as particular cases of the parametric annular interference region.

The statistical techniques used in modeling interference include empirical methods and statistical-physical methods. Empirical approaches fit a mathematical model to measured received signals, without regard to the physical generation mechanisms behind the interference. Statistical-physical models, on the other hand, model interference based on the physical principles that govern the generation and propagation of the interference-causing emissions. Statistical-physical models are thus more widely applicable than empirical models [18], [19].

Statistical-physical modeling of co-channel interference in 
random Poisson interference fields has been extensively studied in literature [17], [20]-[24]. In [20], it was shown that interference from a homogeneous Poisson field of interferers distributed over the entire plane can be modeled using the symmetric alpha stable distribution [25]. This result was later extended to include channel randomness [21] and second-order statistics capturing the temporal dependence [22]. Recently, the authors in [17] investigated extensions for a finite-area field and derived the interference moments. Closed form approximations to the interference distribution, however, were not investigated. In our prior work [23], [24], we presented a unified framework from which we derived the co-channel interference statistics in a Poisson field of interferers distributed on a parametric circular annular region. In this paper, we extend the work in [24] for wider range of interferer topologies and Poisson-Poisson cluster field of interferers.

Other key statistical-physical models for co-channel interference in random Poisson interference fields include Middleton Class A, B, and C models [19]. Middleton models are useful because they characterize a wider range of physical conditions, including narrowband and broadband interference emissions, transients at the receiver, and background thermal noise [18], [19]. Middleton models, however, have not been widely used to characterize co-channel interference in wireless network environments.

Statistical-physical modeling of co-channel interference in random Poisson clustered interference fields was recently studied in [26]. The focus of the work was to characterize the network performance (outage probability and transmission capacity) and the interferer clusters were assumed to be distributed over the entire plane. Closed form interference statistics, however, were not derived.

The problem considered in this paper is also closely related to the problem of deriving the amplitude distribution of shot noise processes [27]. Co-channel interference in a planar network of nodes distributed according to any point process can be modeled as a generalized shot noise process [27], [28]. The shot noise process is studied in detail in [27] and existence of generalized shot noise process for any point process was shown in [28]. Properties of the shot noise processes, such as characteristic function for power-law shot noise process [29], are commonly used to evaluate bounds on outage probabilities in wireless networks [13], [30]. To the best of our knowledge, closed form expression of the amplitude distribution for shot noise process are not known for the interferer topologies considered in this paper.

\section{B. Contribution, Organization, and Notation}

In this paper, we derive the interference statistics from a field of Poisson and Poisson-Poisson clustered distributed interferers. Further, for each of the interferer distributions, we derive the statistics for interferers or interferer clusters distributed over (i) the entire plane, (ii) finite-area annular region, and (iii) infinite-area annular region with a guard zone around the desired receiver. One of the key contributions of this paper is to develop a unified framework to derive the co-channel interference statistics in different wireless network
TABLE I: Summary of Notation

\begin{tabular}{|c|c|}
\hline Symbol & Description \\
\hline $\begin{array}{c}\Pi=\left\{\mathbf{R}_{i}\right\} \\
\mathbf{K} \\
\Gamma \\
\mathbf{R}=\left\|\mathbf{R}-R_{m}\right\| \\
\mathbf{X}=\mathbf{B} e^{j \phi} \\
\gamma>2 \\
\mathbf{g}=\mathbf{h} e^{j \boldsymbol{\theta}} \\
\mathbf{Y}=\mathbf{Y}_{I}+j \mathbf{Y}_{Q} \\
\overline{\mathbf{Y}} \triangleq\left\{\mathbf{Y}_{I}, \mathbf{Y}_{Q}\right\} \\
\bar{\omega}=\left[\omega_{I}, \omega_{Q}\right]^{T} \\
|\bar{\omega}|, \bar{\omega}_{\phi} \\
\Phi=(\bar{\omega}), \Psi_{\overline{\mathbf{Y}}}(\bar{\omega}) \\
\mathbf{\Lambda}(|\bar{\omega}|) \\
\lambda \\
\lambda_{c} \\
\lambda_{f} \\
r_{l}, r_{h} \\
R_{l}, R_{h} \\
\alpha, \sigma \\
A, \Omega_{2 A} \\
p_{l}, \sigma_{l}^{2}\end{array}$ & $\begin{array}{c}\text { point process of active interferers } \\
\text { (random) number of active interferers } \\
\text { region containing interferers } \\
\text { receiver location } \\
\text { (random) distance of interferer from receiver } \\
\text { amplitude and phase of interferer emissions } \\
\text { power pathloss exponent } \\
\text { amplitude and phase of narrowband fading } \\
\text { (complex) sum interference at receiver } \\
\text { inphase and quadrature phase components } \\
\text { frequency variables for characteristic function of } \overline{\mathbf{Y}} \\
\triangleq \sqrt{\omega_{I}^{2}+\omega_{Q}^{2}}, \triangleq-\text { tan }{ }^{-1}\left(\omega_{Q} / \omega_{I}\right) \\
\text { joint characteristic, log-characteristic function of } \overline{\mathbf{Y}} \\
=O\left(|\bar{\omega}|^{4}\right) \text { as }|\bar{\omega}| \rightarrow 0 \text { correction term given by }(18) \\
\text { intensity of } \Pi \text { for a Poisson interferer field } \\
\text { intensity of Poisson process for cluster centers } \\
\text { intensity of Poisson process for nodes in a cluster } \\
\text { inner, outer radii of annular interferer region } \\
\text { inner, outer radii of region with cluster centers } \\
\text { parameters of symmetric alpha stable model } \\
\text { parameters of Middleton Class A model } \\
\text { parameters of Gaussian mixture model, } l \geq 0\end{array}$ \\
\hline
\end{tabular}

environments and establish the applicability of the symmetric alpha stable and Gaussian mixture model (with Middleton Class A model as a particular form). Analytical constraints on the system model parameters for which these distributions accurately model the statistical properties of the interference are also derived. When exact statistics cannot be derived in closed form, the paper focuses on accurately modeling the tail probability of the interference distribution.

The paper is organized as follows. Section II discusses the system model. Section III derives the interference statistics for interferers distributed according to a homogeneous spatial Poisson point process. Section IV derives the interference statistics for a interferers distributed according to a homogeneous spatial Poisson-Poisson clustered process. Section V summarizes the interference models derived in this paper. Section VI presents results from numerical simulations to corroborate our claims. Appendix A contains a brief discussion on the statistical properties of the interference models derived in the paper.

Throughout this paper, random variables are represented using boldface notation, deterministic parameters are represented using non-boldface type, $\mathbb{E}_{\mathbf{X}}\{f(\mathbf{X})\}$ denotes the expectation of the function $f(\mathbf{X})$ with respect to the random variable $\mathbf{X}$, $\mathbb{P}(\cdot)$ denotes the probability of a random event, and $\|\cdot\|$ denotes the Euclidean norm. Table I summarizes the notation used in this paper.

\section{SySTEM MODEL}

At each sampling time instant $n$, the locations of the active interferers are assumed to be distributed according to a homogeneous spatial point process $\Pi=\left\{\mathbf{R}_{1}, \mathbf{R}_{2}, \cdots\right\}$ over the space $\Gamma$, where $\mathbf{R}_{i}$ are the random locations of the interferers. This model is sufficient to capture both the emerging interferers, whose contributions arrive at the receiver for the first time at the time instant $n$, and interferers that first 
emerged at some prior sampling time instant $m<n$ but are still active till the sample time $n$ [22].

The baseband model for the sum interference $\mathbf{Y}$ at any time instant can then be represented as

$$
\mathbf{Y}=\sum_{i=1}^{\mathbf{K}} \mathbf{r}_{i}^{-\frac{\gamma}{2}} \mathbf{g}_{i} \mathbf{X}_{i}
$$

where $\mathbf{K}$ is the random number of active interferers at that time instant, $i$ is the interferer index, $\mathbf{r}_{i}=\left\|\mathbf{R}_{i}-R_{m}\right\|$ are the random distances of active interferers from the receiver, $\gamma$ is the power pathloss exponent, $\mathbf{g}_{i}$ is the independent and identically distributed (i.i.d.) random fast fading experienced by each interferer emission, and $\mathbf{X}_{i}$ are the random interferer emissions.

We assume that all potential interferers have i.i.d. symmetric narrowband emissions of the form [18]

$$
\mathbf{X}_{i}=\mathbf{B}_{i} e^{j \phi_{i}}=\mathbf{B}_{i} \cos \left(\phi_{i}\right)+j \mathbf{B}_{i} \sin \left(\phi_{i}\right)
$$

where $\mathbf{B}_{i}$ is the i.i.d. envelope, and $\phi_{i}$ is the i.i.d. random phase of the emissions. Further, we assume that the emerging times of the interferers are uniformly distributed between the sampling times at the receiver. Thus the phase $\phi_{i}$ of the emissions at the sampling instants can be assumed to be uniformly distributed on $[0,2 \pi]$. The assumption of i.i.d. emissions is valid for wireless communication networks without power control and may not be true for modeling interference from diverse types of interferers with unequal transmit power (e.g. base stations and mobile users).

The fast fading experienced by the interferer emissions is also assumed to be narrowband of the form

$$
\mathbf{g}_{i}=\mathbf{h}_{i} e^{j \boldsymbol{\theta}_{i}}
$$

where $\mathbf{h}_{i}$ is the random amplitude scaling and $\theta_{i}$ is the random phase variation due to fading. The in-phase and quadraturephase components of the emissions are assumed to experience uncorrelated fading and thus $\boldsymbol{\theta}_{i}$ is uniformly distributed on $[0,2 \pi]$. The sum interference can be expressed as

$$
\mathbf{Y}=\sum_{i=1}^{\mathbf{K}} \mathbf{r}_{i}^{-\frac{\gamma}{2}} \mathbf{h}_{i} \mathbf{B}_{i} \cos \left(\boldsymbol{\phi}_{i}+\boldsymbol{\theta}_{i}\right)+j \sum_{i=1}^{\mathbf{K}} \mathbf{r}_{i}^{-\frac{\gamma}{2}} \mathbf{h}_{i} \mathbf{B}_{i} \sin \left(\boldsymbol{\phi}_{i}+\boldsymbol{\theta}_{i}\right)
$$

\section{Co-Channel Interference in a Poisson Field of INTERFERERS}

Consider a scenario, as shown in Fig. 1, in which the spatial point process $\Pi$ in (1) is a homogeneous spatial Poisson point process with intensity $\lambda$ and the interferers are distributed over the space $\Gamma\left(r_{l}, r_{h}\right)$. The parametric interference space is defined as

$$
\Gamma\left(r_{l}, r_{h}\right)=\left\{x \in \mathbb{R}^{2}: r_{l} \leq\|x\| \leq r_{h}\right\} .
$$

From (4), the joint characteristic function of the in-phase and quadrature-phase components of the sum interference $\mathbf{Y}=$ $\mathbf{Y}_{I}+j \mathbf{Y}_{Q}$ can be expressed as

$$
\begin{aligned}
& \Phi_{\mathbf{Y}_{I}, \mathbf{Y}_{Q}}\left(\omega_{I}, \omega_{Q}\right) \\
& =\mathbb{E}_{\mathbf{Y}_{I}, \mathbf{Y}_{Q}}\left\{e^{j \omega_{I} \mathbf{Y}_{I}+j \omega_{Q} \mathbf{Y}_{Q}}\right\}
\end{aligned}
$$

$$
\begin{aligned}
&= \mathbb{E}\left\{e^{j \sum_{i=1}^{\mathrm{K}} \mathbf{r}_{i}^{-\frac{\gamma}{2}} \mathbf{h}_{i} \mathbf{B}_{i}\left(\omega_{I} \cos \left(\boldsymbol{\phi}_{i}+\boldsymbol{\theta}_{i}\right)+\omega_{Q} \sin \left(\boldsymbol{\phi}_{i}+\boldsymbol{\theta}_{i}\right)\right)}\right\} \\
&= \mathbb{E}\left\{e^{j|\bar{\omega}| \sum_{i=1}^{\mathrm{K}} \mathbf{r}_{i}^{-\frac{\gamma}{2}} \mathbf{h}_{i} \mathbf{B}_{i} \cos \left(\boldsymbol{\phi}_{i}+\boldsymbol{\theta}_{i}+\bar{\omega}_{\phi}\right)}\right\} \\
&= \sum_{k=0}^{\infty} \mathbb{E}\left\{e^{j|\bar{\omega}| \sum_{i=1}^{k} \mathbf{r}_{i}^{-\frac{\gamma}{2}} \mathbf{h}_{i} \mathbf{B}_{i} \cos \left(\boldsymbol{\phi}_{i}+\boldsymbol{\theta}_{i}+\bar{\omega}_{\phi}\right)} \mid k \text { in } \Gamma\left(r_{l}, r_{h}\right)\right\} \\
& \times \mathbb{P}\left(k \text { in } \Gamma\left(r_{l}, r_{h}\right)\right)
\end{aligned}
$$

where $\bar{\omega}=\left[\omega_{I}, \omega_{Q}\right]^{T},|\bar{\omega}|=\sqrt{\omega_{I}^{2}+\omega_{Q}^{2}}$, and $\bar{\omega}_{\phi}=$ $-\tan ^{-1}\left(\frac{\omega_{Q}}{\omega_{I}}\right)$. The expectation in (7) is with respect to the set of random variables $\left\{\mathbf{r}_{i}, \mathbf{h}_{i}, \mathbf{B}_{i}, \boldsymbol{\phi}_{i}, \boldsymbol{\theta}_{i}\right\}$.

Conditioned on the number of interferers present in the space $\Gamma\left(r_{l}, r_{h}\right)$, the interferer locations are mutually independent and uniformly distributed across this space [25]. Henceforth, we remove the conditioning on the number of interferers from the expectation by noting that the interferers are uniformly distributed over $\Gamma\left(r_{l}, r_{h}\right)$. Further, in the absence of power control, the interferer emissions can be assumed to be i.i.d.. The characteristic function can then be expressed as

$$
\begin{aligned}
& \Phi_{\overline{\mathbf{Y}}}(\bar{\omega})=\sum_{k=0}^{\infty}\left[\mathbb{E}\left\{e^{j|\bar{\omega}| \mathbf{r}^{-\frac{\gamma}{2}} \mathbf{h B} \cos \left(\boldsymbol{\phi}+\boldsymbol{\theta}+\bar{\omega}_{\phi}\right)}\right\}\right]^{k} \times \\
& \frac{\left[\lambda \pi\left(r_{h}^{2}-r_{l}^{2}\right)\right]^{k} e^{-\lambda \pi\left(r_{h}^{2}-r_{l}^{2}\right)}}{k !} \\
&=e^{\lambda \pi\left(r_{h}^{2}-r_{l}^{2}\right)\left(\mathbb{E}\left\{e^{j|\bar{\omega}| \mathbf{r}-\frac{\gamma}{2} \mathbf{h B} \cos \left(\boldsymbol{\phi}+\boldsymbol{\theta}+\bar{\omega}_{\phi}\right)}\right\}-1\right)}
\end{aligned}
$$

where $\overline{\mathbf{Y}}$ is the set $\left\{\mathbf{Y}_{I}, \mathbf{Y}_{Q}\right\}$. By taking the logarithm of $\Phi \overline{\mathbf{Y}}(\bar{\omega})$, the log-characteristic function is

$$
\begin{aligned}
\psi_{\overline{\mathbf{Y}}}(\bar{\omega}) & \triangleq \log \Phi_{\overline{\mathbf{Y}}}(\bar{\omega}) \\
& =\lambda \pi\left(r_{h}^{2}-r_{l}^{2}\right)\left(\mathbb{E}\left\{e^{j|\bar{\omega}| \mathbf{r}^{-\frac{\gamma}{2}} \mathbf{h B} \cos \left(\boldsymbol{\phi}+\boldsymbol{\theta}+\bar{\omega}_{\phi}\right)}\right\}-1\right) .
\end{aligned}
$$

By using the identity

$$
e^{j a \cos (\phi)}=\sum_{k=0}^{\infty} j^{k} \epsilon_{k} J_{k}(a) \cos (k \phi)
$$

where $\epsilon_{0}=1, \epsilon_{k}=2$ for $k \geq 1$, and $J_{k}(\cdot)$ denotes the Bessel function of order $k$, the log-characteristic function can be expressed as

$$
\begin{array}{r}
\psi_{\overline{\mathbf{Y}}}(\bar{\omega})=\lambda \pi\left(r_{h}^{2}-r_{l}^{2}\right)\left(\mathbb { E } \left\{\sum_{k=0}^{\infty} j^{k} \epsilon_{k} J_{k}\left(|\bar{\omega}| \mathbf{r}^{-\frac{\gamma}{2}} \mathbf{h B}\right) \times\right.\right. \\
\left.\left.\cos \left(k\left(\boldsymbol{\phi}+\boldsymbol{\theta}+\bar{\omega}_{\phi}\right)\right)\right\}-1\right)
\end{array}
$$

Since $\phi$ and $\boldsymbol{\theta}$ are assumed to be uniformly distributed on $[0,2 \pi], \mathbb{E}_{\boldsymbol{\phi}, \boldsymbol{\theta}}\left\{\cos \left(k\left(\boldsymbol{\phi}+\boldsymbol{\theta}+\bar{\omega}_{\phi}\right)\right)\right\}=0$ for $k \geq 1$, and (12) reduces to

$$
\psi_{\overline{\mathbf{Y}}}(\bar{\omega})=\lambda \pi\left(r_{h}^{2}-r_{l}^{2}\right)\left(\mathbb{E}_{\mathbf{r}, \mathbf{h}, \mathbf{B}}\left\{J_{0}\left(|\bar{\omega}| \mathbf{r}^{-\frac{\gamma}{2}} \mathbf{h B}\right)\right\}-1\right) .
$$

The log-characteristic function derived in (13) holds in general for narrowband interferers distributed over the parametric space $\Gamma\left(r_{l}, r_{h}\right)$, governed by the parameters $r_{h}$ and $r_{l}$ and 
Area containing Interferers

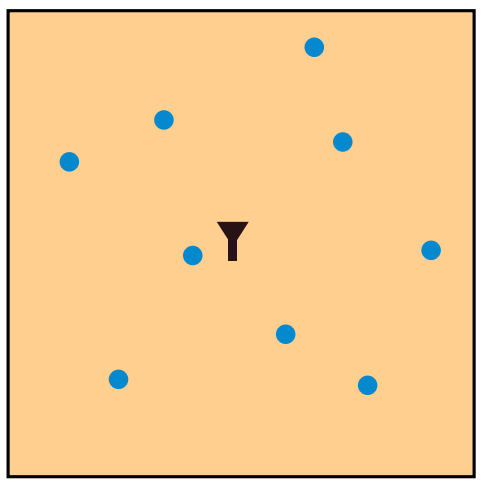

Case I: Entire Plane

$r_{l}=0, r_{h} \rightarrow \infty$
Y Receiver Location Snapshot of Interferer Locations

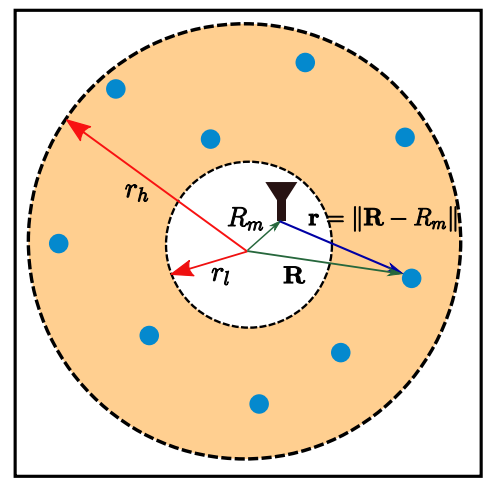

Case II: Finite-area Annular Region

$0 \leq r_{l}<r_{h}<\infty, R_{m} \notin \Gamma\left(r_{l}, r_{h}\right)$

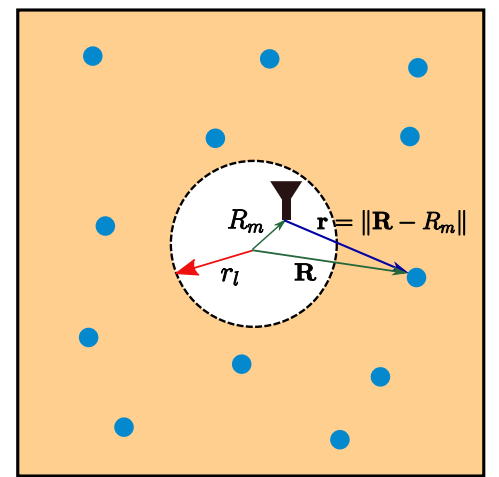

Case III: Infinite-area with Guard Zone

$r_{l}>0, r_{h} \rightarrow \infty, R_{m} \notin \Gamma\left(r_{l}, r_{h}\right)$

Fig. 1: Interference space and receiver location for different network topologies in a field of Poisson distributed interferers categorized by the region containing the interferers.

the receiver location $R_{m}$. The receiver location $R_{m}$ affects the expectation in (13). We now consider the following three cases and further simplify the log-characteristic function.

\section{A. Case I: Interferers distributed over the entire plane $\left(r_{l}=\right.$ $\left.0, r_{h} \rightarrow \infty\right)$}

Consider a wireless network, as shown in Fig. 1, where the interfering sources are distributed according to a spatial Poisson point process over the entire plane. Note that $\left\|R_{m}\right\|$ can be assumed to be zero without any loss in generality of the result. This scenario corresponds to a decentralized network in which nodes do not employ any contention-based MAC protocol, and has been widely studied [3], [9], [20][24]. We consider the interference space $\Gamma\left(0, r_{h}\right)$ and take the limit on the log-characteristic function as $r_{h} \rightarrow \infty$ [9], [20]. Recall that the expectation in (13) is conditioned such that the interferer locations are uniformly distributed over $\Gamma\left(r_{l}, r_{h}\right)$. The distance of each interferer from the receiver thus follows the distribution

$$
f_{\mathbf{r} \mid \mathbf{K}}(r \mid K)= \begin{cases}\frac{2 r}{r_{h}^{2}} & \text { if } 0 \leq r \leq r_{h} \\ 0 & \text { otherwise }\end{cases}
$$

Expanding the expectation in (13), we have

$$
\begin{array}{r}
\psi_{\overline{\mathbf{Y}}}(\bar{\omega})=\lim _{r_{h} \rightarrow \infty} \lambda \pi r_{h}^{2}\left(\int_{0}^{r_{h}} \mathbb{E}_{\mathbf{h}, \mathbf{B}}\left\{J_{0}\left(|\bar{\omega}| r^{-\frac{\gamma}{2}} \mathbf{h B}\right)\right\} \frac{2 r}{r_{h}^{2}} d r\right. \\
-1) .
\end{array}
$$

Integrating the above by parts, noting that $\lim _{r_{h} \rightarrow \infty} \mathbb{E}_{\mathbf{h}, \mathbf{B}}\left\{r_{h}^{2}\left(J_{0}\left(|\bar{\omega}| r_{h}^{-\frac{\gamma}{2}} \mathbf{h B}\right)-1\right)^{\prime}\right\}=0$ for $\gamma>2$, and $\frac{d}{d x} J_{0}(x)=-J_{1}(x)$, we have

$$
\psi_{\overline{\mathbf{Y}}}(\bar{\omega})=-|\bar{\omega}|^{\frac{4}{\gamma}} \lambda \pi \mathbb{E}_{\mathbf{h}, \mathbf{B}}\left\{\mathbf{h}^{\frac{4}{\gamma}} \mathbf{B}^{\frac{4}{\gamma}}\right\} \int_{0}^{\infty} \frac{J_{1}(x)}{x^{\frac{4}{\gamma}}} d x .
$$

Equation (15) is the log-characteristic function of an isotropic symmetric alpha stable distribution centered at zero such that

$$
\psi_{\mathbf{Y}_{I}, \mathbf{Y}_{Q}}\left(\omega_{I}, \omega_{Q}\right)=-\sigma\left|\sqrt{\omega_{I}^{2}+\omega_{Q}^{2}}\right|^{\alpha}
$$

where $\alpha=\frac{4}{\gamma}$ is the characteristic exponent $(0<\alpha<2)$, and $\sigma=\lambda \pi \mathbb{E}_{\mathbf{h}, \mathbf{B}}\left\{\mathbf{h}^{\alpha} \mathbf{B}^{\alpha}\right\} \int_{0}^{\infty} \frac{J_{1}(x)}{x^{\alpha}} d x$ is the dispersion parameter $(\sigma>0)$ of the symmetric alpha stable distribution [25]. Hence, the sum interference in a Poisson field of interferers distributed over the entire plane follows a symmetric alpha stable distribution.

\section{B. Case II: Interferers distributed over a finite-area annular region $\left(0 \leq r_{l}<r_{h}<\infty, R_{m} \notin \Gamma\left(r_{l}, r_{h}\right)\right)$}

Consider a wireless network, as shown in Fig. 1, where the interferers are distributed over a finite-area annular region. When $r_{l}>0$ and $\left\|R_{m}\right\|<r_{l}$, this corresponds to a scenario where all the interferers are outside a guard zone around the receiver and within a maximum distance $\left(r_{h}<\infty\right)$ beyond which they do not generate significant interference. When $\left\|R_{m}\right\|>r_{h}$, this corresponds to a scenario where the interferers are distributed over a finite-area circular or annular region with the receiver exterior to this region. The former scenario is applicable for wireless networks with contentionbased or scheduling-based MAC protocols creating a guard zone around the receiver (e.g. cellular networks with reuse factor of one and ad hoc networks with guard zones [15]). The latter scenario is useful in characterizing the interference from a hotspot (e.g. interferers localized in space around a cafe) and in cellular networks with reuse factor greater than one. In cellular networks with reuse factor greater than one, the interferers are distributed within a regular pattern of isolated cell sites and the sum interference is thus a sum of the interference from these isolated finite-area cell sites.

In [18], Middleton proposed an approximation of the logcharacteristic function for $|\bar{\omega}|$ in the neighborhood of zero. From Fourier analysis, the behavior of the characteristic function for $|\bar{\omega}|$ in the neighborhood of zero governs the tail prob- 
ability of the random envelope. The proposed approximation is based on the following identity [18]:

$$
\begin{aligned}
& \mathbb{E}_{\mathbf{r}, \mathbf{h}, \mathbf{B}}\left\{J_{0}\left(|\bar{\omega}| \mathbf{r}^{-\frac{\gamma}{2}} \mathbf{h B}\right)\right\}=e^{-\frac{|\bar{\omega}|^{2} \mathbb{E}_{\mathbf{r}, \mathbf{h}, \mathbf{B}}\left\{\mathbf{r}^{-\gamma} \mathbf{h}^{2} \mathbf{B}^{2}\right\}}{4}} \times \\
& (1+\boldsymbol{\Lambda}(|\bar{\omega}|))
\end{aligned}
$$

where $\boldsymbol{\Lambda}(|\bar{\omega}|)$ ) indicates a correction term with the lowest exponent in $|\bar{\omega}|$ of four and is given by

$\boldsymbol{\Lambda}(|\bar{\omega}|))=\sum_{k=2}^{\infty} \frac{\left(\mathbb{E}_{\mathbf{Z}}\{\mathbf{Z}\}\right)^{k}|\bar{\omega}|^{2 k}}{2^{2 k} k !} \mathbb{E}_{\mathbf{Z}}\left\{{ }_{1} F_{1}\left(-k ; 1 ; \frac{\mathbf{Z}}{\mathbb{E}_{\mathbf{Z}}\{\mathbf{Z}\}}\right)\right\}$

where the random variable $\mathbf{Z}=\mathbf{r}^{-\gamma} \mathbf{h}^{2} \mathbf{B}^{2}$, and ${ }_{1} F_{1}(a ; b ; x)$ is the confluent hypergeometric function of the first kind, such that $\boldsymbol{\Lambda}(|\bar{\omega}|)=O\left(|\bar{\omega}|^{4}\right)$ as $|\bar{\omega}| \rightarrow 0$.

Using this identity, and approximating $\Lambda(|\bar{\omega}|)<<1$ for $|\bar{\omega}|$ in the neighborhood of zero, the log-characteristic function in (13) can be expressed as

$$
\psi_{\overline{\mathbf{Y}}}(\bar{\omega}) \approx \lambda \pi\left(r_{h}^{2}-r_{l}^{2}\right)\left(e^{-\frac{|\bar{\omega}|^{2} \mathbb{E}_{\mathbf{r}, \mathbf{h}, \mathbf{B}}\left\{\mathbf{r}^{-\gamma} \mathbf{h}^{2} \mathbf{B}^{2}\right\}}{4}}-1\right) .
$$

Equation (19) is the log-characteristic function of a Middleton Class A distribution such that

$$
\psi_{\mathbf{Y}_{I}, \mathbf{Y}_{Q}}\left(\omega_{I}, \omega_{Q}\right)=A\left(e^{-\frac{\left(\omega_{I}^{2}+\omega_{Q}^{2}\right) \Omega_{2 A}}{2 A}}-1\right)
$$

where $A=\lambda \pi\left(r_{h}^{2}-r_{l}^{2}\right)$ is the overlap index that indicates the amount of impulsiveness of the interference, and $\Omega_{2 A}=\frac{A \times \mathbb{E}_{\mathbf{r}, \mathbf{h}, \mathbf{B}}\left\{\mathbf{r}^{-\gamma} \mathbf{h}^{2} \mathbf{B}^{2}\right\}}{2}$ is the mean intensity of the interference [19]. Hence, the co-channel interference from a field of Poisson distributed interferers over the finite-area space $\Gamma\left(r_{l}, r_{h}\right)$ with $R_{m} \notin \Gamma\left(r_{l}, r_{h}\right)$ follows the Middleton Class A distribution. It should be emphasized that the correspondence to the Middleton Class A distribution is particularly valid for modeling the tail probabilities.

The approximation in (17) and the subsequent interference model in (20) is valid for $R_{m} \notin \Gamma\left(r_{l}, r_{h}\right)$, since $\Omega_{2 A} \rightarrow \infty$ as $\left\|R_{m}\right\| \rightarrow r_{l}$ or as $\left\|R_{m}\right\| \rightarrow r_{h}$. This is unlike Case $I$ in Section III-A where the interference was modeled for $r_{l}=0$. This is the key difference between the symmetric alpha stable and Middleton Class A models for interference.

Next, we quantify the range of the system model parameters over which the Middleton Class A model provides an accurate approximation to the co-channel interference in this scenario. From (17), a first-order measure of the accuracy of the approximation can be expressed by comparing the coefficient of $|\bar{\omega}|^{4}$ term in $e^{-\frac{|\bar{\omega}|^{2} \mathbb{E}_{\mathbf{r}, \mathbf{h}, \mathbf{B}}\left\{\mathbf{r}^{\left.-\gamma_{\mathbf{h}} \mathbf{B}^{2}\right\}}\right.}{4}}$ against the coefficient of $|\bar{\omega}|^{4}$ in the correction term $\boldsymbol{\Lambda}(|\bar{\omega}|)$. Using the fact that

$$
{ }_{1} F_{1}(-2 ; 1 ; x)=\frac{1}{2}\left(x^{2}-4 x+2\right),
$$

the coefficient of $|\bar{\omega}|^{4}$ in the correction term (i.e., $c_{4}$ ) can be expressed as

$$
c_{4}=\frac{\mathbb{E}_{\mathbf{Z}}\left\{\mathbf{Z}^{2}\right\}-2\left[\mathbb{E}_{\mathbf{Z}}\{\mathbf{Z}\}\right]^{2}}{128} .
$$

Thus, the Middleton Class A model provides a good approximation when the system parameters, such as $r_{h}, r_{l}, R_{m}$, and $\gamma$, satisfy

$$
\Rightarrow\left|\frac{\frac{\mathbb{E}_{\mathbf{Z}}\left\{\mathbf{Z}^{2}\right\}-2\left[\mathbb{E}_{\mathbf{Z}}\{\mathbf{Z}\}\right]^{2}}{128} \mid<<\frac{\left[\mathbb{E}_{\mathbf{Z}}\{\mathbf{Z}\}\right]^{2}}{32}}{4 \times\left[\mathbb{E}_{\mathbf{r}, \mathbf{h}, \mathbf{B}}\left\{\mathbf{r}^{-\gamma} \mathbf{h}^{2} \mathbf{B}^{2}\right\}\right]^{2}}-\frac{1}{2}\right|<<1 .
$$

To provide some intuition about the above result, for a nonrandom $\mathbf{h}$ and $\mathbf{B}$, the condition is satisfied when $\left\|R_{m}\right\|<<r_{l}$ and $\frac{r_{l}}{r_{h}}$ is greater than a fraction that depends on $\gamma$ and $R_{m}$, or when $\left\|R_{m}\right\|>>r_{h}$. The conditions $\left\|R_{m}\right\|<<r_{l}$ and $\left\|R_{m}\right\|>>r_{h}$ ensure that the interferers are not close to the receiver and a lower bound on $\frac{r_{l}}{r_{h}}$ ensures that $r_{h}$ is not very large compared to $r_{l}$ when $\left\|R_{m}\right\|<r_{l}$.

\section{Case III: Interferers distributed over infinite-area annular region with guard zone $\left(r_{l}>0, r_{h} \rightarrow \infty\right.$, and $\left.\left\|R_{m}\right\|<r_{l}\right)$}

Consider a wireless network, as shown in Fig. 1, where the interfering sources are distributed according to a spatial Poisson point process on the entire plane, except within a guard zone around the receiver. The applicability of Case II for guard zone scenarios was limited to finite-area fields and does provide a good approximation for a wide range of system parameters. In this subsection, we allow the interference region to have infinite area and is thereby more applicable to large random wireless networks with guard zones [15]. We consider the interference space $\Gamma\left(r_{l}, r_{h}\right)$ and take the limit on the logcharacteristic function as $r_{h} \rightarrow \infty$. Conditioned on the number of interferers in $\Gamma\left(r_{l}, r_{h}\right)$, the interferer locations are mutually independent and uniformly distributed in the space $\Gamma\left(r_{l}, r_{h}\right)$. Thus as $r_{h} \rightarrow \infty$, with high probability, the distance of an interferer from receiver located at $R_{m}$ can be approximated as $\mathbf{r}=\left\|\mathbf{R}-R_{m}\right\| \approx\|\mathbf{R}\|$, particularly for $\left\|R_{m}\right\|<<r_{l}$. The distance of each interferer from the receiver thus follows the distribution

$$
f_{\mathbf{r} \mid \mathbf{K}}(r \mid K)= \begin{cases}\frac{2 r}{r_{h}^{2}-r_{l}^{2}} & \text { if } r_{l} \leq r \leq r_{h} \\ 0 & \text { otherwise }\end{cases}
$$

Expanding the expectation in (13), we have

$$
\begin{array}{r}
\psi_{\overline{\mathbf{Y}}}(\bar{\omega})=\lim _{r_{h} \rightarrow \infty} \lambda \pi\left(r_{h}^{2}-r_{l}^{2}\right)\left(\int_{r_{l}}^{r_{h}} \mathbb{E}_{\mathbf{h}, \mathbf{B}}\left\{J_{0}\left(|\bar{\omega}| r^{-\frac{\gamma}{2}} \mathbf{h B}\right)\right\} \times\right. \\
\left.\frac{2 r}{r_{h}^{2}-r_{l}^{2}} d r-1\right) .
\end{array}
$$

Integrating the above by parts, reordering terms, and noting that $\lim _{r_{h} \rightarrow \infty} \lambda \pi r_{h}^{2}\left(\mathbb{E}_{\mathbf{h}, \mathbf{B}}\left\{J_{0}\left(|\bar{\omega}| r_{h}^{-\frac{\gamma}{2}} \mathbf{h B}\right)\right\}-1\right)=0$ for $\gamma>$ 2 , we have

$$
\begin{gathered}
\psi_{\overline{\mathbf{Y}}}(\bar{\omega})=-\lambda \pi r_{l}^{2}\left(\mathbb{E}_{\mathbf{h}, \mathbf{B}}\left\{J_{0}\left(|\bar{\omega}| r_{l}^{-\frac{\gamma}{2}} \mathbf{h B}\right)\right\}-1\right)- \\
\lim _{r_{h} \rightarrow \infty} \lambda \pi \int_{r_{l}}^{r_{h}} \frac{\partial}{\partial r}\left(\mathbb{E}_{\mathbf{h}, \mathbf{B}}\left\{J_{0}\left(|\bar{\omega}| r^{-\frac{\gamma}{2}} \mathbf{h B}\right)\right\}\right) r^{2} d r .
\end{gathered}
$$


Invoking the identity (17), and approximating $\Lambda(|\bar{\omega}|)<<1$ for $|\bar{\omega}|$ in the neighborhood of zero, the log-characteristic function can be expressed as

$$
\begin{aligned}
& \psi_{\overline{\mathbf{Y}}}(\bar{\omega}) \approx-\lambda \pi r_{l}^{2}\left(e^{-\frac{|\bar{\omega}|^{2} r_{l}^{-}-\bar{E}_{\mathbf{h}, \mathbf{B}}\left\{\mathbf{h}^{2} \mathbf{B}^{2}\right\}}{4}}-1\right)-
\end{aligned}
$$

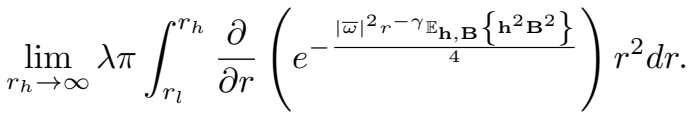

Note that unlike (17), the approximation in (27) involves a non-random $r$. Using Taylor series expansion of $e^{x}$, the $\log$ characteristic function reduces to

$\psi_{\overline{\mathbf{Y}}}(\bar{\omega})=\lambda \pi r_{l}^{2}\left[\sum_{k=1}^{\infty} \frac{(-1)^{k}|\bar{\omega}|^{2 k}}{4^{k} k !}\left(\mathbb{E}\left\{\mathbf{h}^{2} \mathbf{B}^{2}\right\}\right)^{k} r_{l}^{-\gamma k} \frac{2}{k \gamma-2}\right]$

valid for $\gamma>2$. The $\frac{2}{k \gamma-2}$ multiplicative factor inside the summation prevents the log-characteristic function to be expressed in closed form. We thus approximate the function $\frac{2}{k \gamma-2}$ as $\eta e^{\beta k}$ for $k \geq 1$. The parameters $\eta$ and $\beta$ are chosen to minimize the weighted mean squared error (WMSE)

$$
\{\eta, \beta\}=\arg \min _{\eta, \beta} \sum_{k=1}^{\infty}\left(\frac{2}{k \gamma-2}-\eta e^{\beta k}\right)^{2} u(k)
$$

where $u(k)$ are the weights. The weights should be chosen such that penalty of error is large when $k$ is small, since it affects the coefficients of terms with lower order exponents of $|\bar{\omega}|$. Equation (29) is an unconstrained nonlinear optimization problem and can be solved efficiently using numerical techniques such as quasi-Newton methods [31]. QuasiNewton methods have superlinear convergence and require $O(\ln (|\ln (\epsilon)|))$ number of iterations and $O\left(d^{2} \ln (|\ln (\epsilon)|)\right)$ algebraic computational effort, where $d$ is the dimensionality of the problem and $\epsilon$ is the maximum permissible error tolerance in the result. Table II lists the values for $\{\eta, \beta\}$ and the associated WMSE for certain values of $\gamma$, using the weights $u(k)=e^{-k}$. By approximating $\frac{2}{k \gamma-2}$ as $\eta e^{\beta k}$ for $k \geq 1$, the log-characteristic exponent can be expressed as

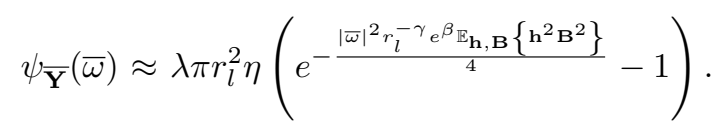

Equation (30) is the log-characteristic function of a Middleton Class A distribution such that

$$
\psi_{\mathbf{Y}_{I}, \mathbf{Y}_{Q}}\left(\omega_{I}, \omega_{Q}\right)=A\left(e^{-\frac{\left(\omega_{I}^{2}+\omega_{Q}^{2}\right) \Omega_{2 A}}{2 A}}-1\right)
$$

where $A=\lambda \pi r_{l}^{2} \eta$ is the overlap index that indicates the impulsiveness of the interference, and $\Omega_{2 A}=$ $\frac{A \times r_{l}^{-\gamma} e^{\beta} \mathbb{E}_{\mathbf{h}, \mathbf{B}}\left\{\mathbf{h}^{2} \mathbf{B}^{2}\right\}}{2}$ is the mean intensity of the interference [19].

The functional form of $\eta e^{\beta k}$ to approximate $\frac{2}{k \gamma-2}$ for $k \geq 1$ was chosen since, a) it provides a good approximation and enables the log-characteristic function to be expressed in closed form, and b) provides two parameters $\{\eta, \beta\}$ such that $\eta$ affects only the impulsive index $A$, while $\beta$ affects only the variance $\sigma_{m}^{2}=\frac{m}{A} \Omega_{2 A}$ of individual components of the Gaussian mixture form of Middleton Class A model.
TABLE II: Values for $\{\eta, \beta\}$ and the associated weighted mean squared error (WMSE), obtained by solving (29), for different values of the power pathloss exponent $(\gamma)$ and using the weighting function $u(k)=e^{-k}$. Solution to (29) was obtained by using the fminunc function in MATLAB, which uses the BFGS quasi-Newton method [31].

\begin{tabular}{|c|c|c|}
\hline$\gamma$ & $\{\eta, \beta\}$ & WMSE \\
\hline \hline 2.5 & $\{22.818,-1.741\}$ & $4.32 \times 10^{-3}$ \\
\hline 3.0 & $\{7.484,-1.321\}$ & $1.84 \times 10^{-3}$ \\
\hline 3.5 & $\{4.132,-1.132\}$ & $9.81 \times 10^{-4}$ \\
\hline 4.0 & $\{2.781,-1.025\}$ & $5.96 \times 10^{-4}$ \\
\hline 4.5 & $\{2.073,-0.954\}$ & $3.96 \times 10^{-4}$ \\
\hline 5.0 & $\{1.645,-0.905\}$ & $2.80 \times 10^{-4}$ \\
\hline
\end{tabular}

Similar to Case II, a first-order measure of accuracy of the approximation can be expressed by comparing the coefficient of $|\bar{\omega}|^{4}$ term in the true log-characteristic function (26) against the the coefficient of $|\bar{\omega}|^{4}$ term in the approximated logcharacteristic function (30). The two approximations involved are using $\eta e^{\beta k}$ to approximate the function $\frac{2}{k \gamma-2}$ for $k \geq 1$, and approximating $\boldsymbol{\Lambda}(|\bar{\omega}|)<<1$ for $|\bar{\omega}|$ close to zero. Note that the lowest order term affected by the former approximation is the coefficient of $|\bar{\omega}|^{2}$ term. We assume, however, that the approximation error is negligible due to the optimization in (29). Using (17) and (18), the coefficient of $|\bar{\omega}|^{4}$ term in the true log-characteristic function (30) is

$$
\lambda \pi r_{l}^{-2 \gamma+2}\left(\frac{\mathbb{E}\left\{\mathbf{Z}^{2}\right\}+2[\mathbb{E}\{\mathbf{Z}\}]^{2}}{128}\right)\left(\frac{2}{2 \gamma-2}\right)
$$

where $\mathbf{Z}=\mathbf{h}^{2} \mathbf{B}^{2}$. Comparing with the coefficient of $|\bar{\omega}|^{4}$ term in (30), the Middleton Class A distribution provides a good approximation to co-channel interference statistics in this scenario when

$$
\begin{array}{r}
\left|\left(\frac{[\mathbb{E}\{\mathbf{Z}\}]^{2}}{64}\right)\left(\frac{2}{2 \gamma-2}-2 \eta e^{2 \beta}\right)+\left(\frac{\mathbb{E}\left\{\mathbf{Z}^{2}\right\}}{128}\right)\left(\frac{2}{2 \gamma-2}\right)\right| \\
<<\left|\frac{[\mathbb{E}\{\mathbf{Z}\}]^{2}}{32} \eta e^{2 \beta}\right|
\end{array}
$$

Note that if $\eta e^{2 \beta}=\frac{2}{2 \gamma-2}$, then the above condition is same as the one obtained for Case II in (23), with the exception that $\mathbf{Z}=\mathbf{h}^{2} \mathbf{B}^{2}$ in this case. The above condition is independent of the parameter $r_{l}$ that governs the interference space and is valid when the variance of $\mathbf{h}^{2} \mathbf{B}^{2}$ is low when compared to $\left[\mathbb{E}\left\{\mathbf{h}^{2} \mathbf{B}^{2}\right\}\right]^{2}$. The above condition does not capture the error due to the approximation $\mathbf{r}=\left\|\mathbf{R}-R_{m}\right\| \approx \mathbf{R}$, which is true with high probability in this scenario and is particularly valid for $\left\|R_{m}\right\|<<r_{l}$.

\section{Co-Channel Interference in a Poisson-Poisson Cluster Field OF INTERFERERS}

Consider a scenario, as shown in Fig. 2, where the interferers are clustered in space. The center of the clusters are assumed to distributed according to a spatial Poisson point process $\Pi_{c}$ with intensity $\lambda_{c}$ over the space $\Gamma\left(R_{l}, R_{h}\right)$. For each cluster center $\mathbf{R}_{c} \in \Pi_{c}$, interferers are assumed to be distributed according to an independent spatial Poisson process $\Pi_{c, f}$ with intensity $\lambda_{f}$ over the space $\Gamma\left(r_{l}, r_{h}\right)$ around the 


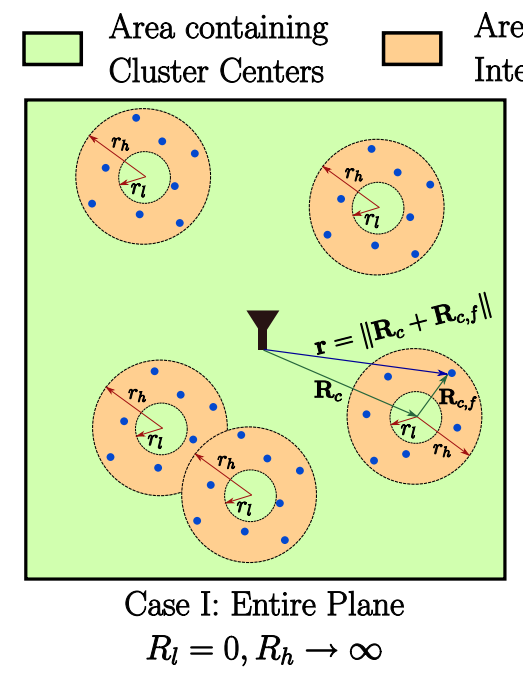

Area containing
Interferers

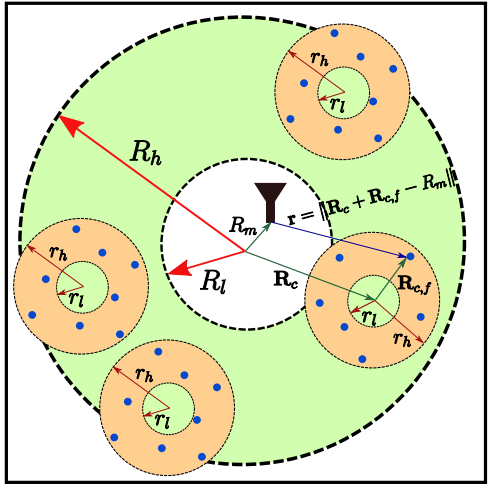

Case II: Finite-area Annular Region

$0 \leq R_{l}<R_{h}<\infty$,

$R_{m} \notin \Gamma\left(R_{l}-r_{h}, R_{h}+r_{h}\right)$
- Snapshot of Interferer Locations

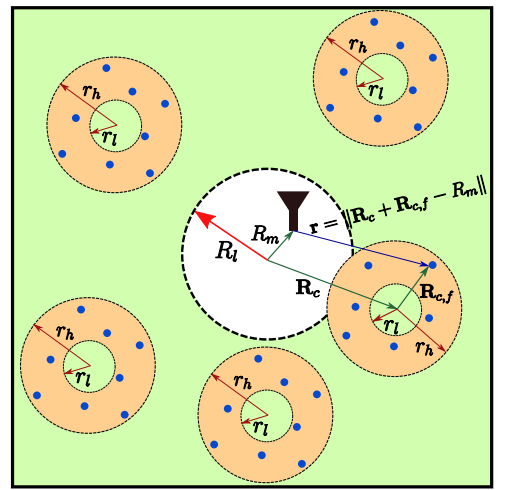

Case III: Infinite-area with Guard Zone

$R_{l}>0, R_{h} \rightarrow \infty, R_{m} \notin \Gamma\left(R_{l}-r_{h}, \infty\right)$

Fig. 2: Interference space and receiver location for different network topologies in a field of Poisson-Poisson cluster distributed interferers categorized by the region containing the cluster centers.

center $\mathbf{R}_{c}$. The point process $\Pi$ in (1) is then a homogeneous spatial Poisson-Poisson cluster process such that

$$
\Pi=\bigcup_{\mathbf{R}_{c} \in \Pi_{c}} \bigcup_{\mathbf{R}_{c, f} \in \Pi_{c, f}}\left\{\mathbf{R}_{c}+\mathbf{R}_{c, f}\right\} .
$$

Note that the cluster centers are themselves not included. The parametric interference space $\Gamma(\cdot, \cdot)$ is defined in (5). When $r_{l}=0, \Pi$ is a Matern cluster process [32].

The joint characteristic function of the in-phase and quadrature-phase components of the sum interference $\mathbf{Y}=$ $\mathbf{Y}_{I}+j \mathbf{Y}_{Q}$ can be expressed as

$$
\begin{aligned}
& \Phi_{\mathbf{Y}_{I}, \mathbf{Y}_{Q}}\left(\omega_{I}, \omega_{Q}\right) \\
& =\mathbb{E}_{\mathbf{Y}_{I}, \mathbf{Y}_{Q}}\left\{e^{j \omega_{I} \mathbf{Y}_{I}+j \omega_{Q} \mathbf{Y}_{Q}}\right\} \\
& =\mathbb{E}\left\{e^{j|\bar{\omega}| \sum_{i=1}^{\mathbf{K}_{c}} \sum_{m=1}^{\mathbf{K}_{c, f}} \mathbf{r}_{i, m}^{-\frac{\gamma}{2}} \mathbf{h}_{i, m} \mathbf{B}_{i, m} \cos \left(\boldsymbol{\phi}_{i, m}+\boldsymbol{\theta}_{i, m}+\bar{\omega}_{\phi}\right)}\right\} \\
& =\sum_{k_{c}=0}^{\infty} \mathbb{E}\left\{e^{j|\bar{\omega}| \sum_{i=1}^{k_{c}} \sum_{m=1}^{\mathbf{K}_{c, f}} \mathbf{r}_{i, m}^{-\frac{\gamma}{2}} \mathbf{h}_{i, m} \mathbf{B}_{i, m} \cos \left(\boldsymbol{\phi}_{i, m}+\boldsymbol{\theta}_{i, m}+\bar{\omega}_{\phi}\right)}\right. \\
& \left.\quad \mid k_{c} \text { in } \Gamma\left(R_{l}, R_{h}\right)\right\} \times \mathbb{P}\left(k_{c} \text { in } \Gamma\left(R_{l}, R_{h}\right)\right)
\end{aligned}
$$

where $\mathbf{K}_{c}$ is the random number of active clusters, $\mathbf{K}_{c, f}$ is the random number of active interferers per cluster, $\bar{\omega}=$ $\left[\omega_{I}, \omega_{Q}\right]^{T},|\bar{\omega}|=\sqrt{\omega_{I}^{2}+\omega_{Q}^{2}}$, and $\bar{\omega}_{\phi}=-\tan ^{-1}\left(\frac{\omega_{Q}}{\omega_{I}}\right)$. The expectation in (34) is with respect to the set of random variables $\left\{\mathbf{K}_{c, f}, \mathbf{r}_{i, m}, \mathbf{h}_{i, m}, \mathbf{B}_{i, m}, \boldsymbol{\phi}_{i, m}, \boldsymbol{\theta}_{i, m}\right\}$. The indexing $(\cdot)_{i, m}$ denotes the $m^{t h}$ active interferer in the $i^{\text {th }}$ cluster.

Conditioned on the number of clusters present in the space $\Gamma\left(R_{l}, R_{h}\right)$, location of the cluster centers $\left(\mathbf{R}_{c}\right)$ are mutually independent and uniformly distributed over this space [25]. Further, in the absence of power control, the sum interference from each cluster can be assumed to be i.i.d., such that

$$
\begin{aligned}
\Phi_{\overline{\mathbf{Y}}}(\bar{\omega})=\sum_{k_{c}=0}^{\infty} & {\left[\mathbb{E}\left\{e^{j|\bar{\omega}| \sum_{m=0}^{\mathbf{K}_{c, f}} \mathbf{r}_{m}^{-\frac{\gamma}{2}} \mathbf{h}_{m} \mathbf{B}_{m} \cos \left(\boldsymbol{\phi}_{m}+\boldsymbol{\theta}_{m}+\bar{\omega}_{\phi}\right)}\right\}\right]^{k_{c}} } \\
& \times \frac{\left[\lambda_{c} \pi\left(R_{h}^{2}-R_{l}^{2}\right)\right]^{k_{c}} e^{-\lambda_{c} \pi\left(R_{h}^{2}-R_{l}^{2}\right)}}{k_{c} !}
\end{aligned}
$$

$$
=e^{A_{c}\left(\mathbb{E}\left\{e^{j|\bar{\omega}| \sum_{m=0}^{\mathbf{K}_{c, f}} \mathbf{r}_{m}^{-\frac{\gamma}{2}} \mathbf{h}_{m} \mathbf{B}_{m} \cos \left(\boldsymbol{\phi}_{m}+\boldsymbol{\theta}_{m}+\overline{\boldsymbol{\omega}}_{\phi}\right)}\right\}-1\right)}
$$

where $\overline{\mathbf{Y}}$ is the set $\left\{\mathbf{Y}_{I}, \mathbf{Y}_{Q}\right\}$, and $A_{c}=\lambda_{c} \pi\left(R_{h}^{2}-R_{l}^{2}\right)$. The expectation in (36) is with respect to the set of random variables $\left\{\mathbf{R}_{c}, \mathbf{K}_{c, f}, \mathbf{R}_{c, m}, \mathbf{h}_{m}, \mathbf{B}_{m}, \boldsymbol{\phi}_{m}, \boldsymbol{\theta}_{m}\right\}$. By taking the logarithm of $\Phi_{\overline{\mathbf{Y}}}(\bar{\omega})$, the log-characteristic function is

$$
\begin{aligned}
& \psi_{\overline{\mathbf{Y}}}(\bar{\omega})= \\
& A_{c}\left(\mathbb{E}\left\{e^{j|\bar{\omega}| \sum_{m=0}^{\mathbf{K}_{c, f}} \mathbf{r}_{m}^{-\frac{\gamma}{2}} \mathbf{h}_{m} \mathbf{B}_{m} \cos \left(\boldsymbol{\phi}_{m}+\boldsymbol{\theta}_{m}+\bar{\omega}_{\phi}\right)}\right\}-1\right) .
\end{aligned}
$$

The above equation can be expressed in the form

$$
\psi_{\overline{\mathbf{Y}}}(\bar{\omega})=A_{c}\left(\mathbb{E}_{\mathbf{R}_{c}}\left\{\mathbb{E}_{\mathbf{Y}_{c, f}}\left\{e^{j|\bar{\omega}| \mathbf{Y}_{c, f}}\right\}\right\}-1\right)
$$

where $\mathbf{Y}_{c, f}$ is the sum interference from an interferer cluster and is a function of the set of random variables $\left\{\mathbf{K}_{c, f}, \mathbf{R}_{c, m}, \mathbf{h}_{m}, \mathbf{B}_{m}, \boldsymbol{\phi}_{m}, \boldsymbol{\theta}_{m}\right\}$, similar to (6). Thus $\mathbf{Y}_{c, f}$ is the sum interference from a field of Poisson distributed interferers over the interference space $\Gamma\left(r_{l}, r_{h}\right)$ around the cluster center $\mathbf{R}_{c}$. Using (13), the log-characteristic function can then be expressed as

$\psi_{\overline{\mathbf{Y}}}(\bar{\omega})=A_{c}\left[\mathbb{E}_{\mathbf{R}_{c}}\left\{e^{A_{f}\left(\mathbb{E}_{\mathbf{R}_{c, f}, \mathbf{h}, \mathbf{B}}\left\{J_{0}\left(|\bar{\omega}| \mathbf{r}^{-\frac{\gamma}{2}} \mathbf{h B}\right)\right\}-1\right)}\right\}-1\right]$

where $A_{f}=\lambda_{f} \pi\left(r_{h}^{2}-r_{l}^{2}\right), \mathbf{r}=\left\|\mathbf{R}_{c}+\mathbf{R}_{c, f}-R_{m}\right\|, \mathbf{R}_{c}$ is uniformly distributed in $\Gamma\left(R_{l}, R_{h}\right)$, and $\mathbf{R}_{c, f}$ is uniformly distributed in $\Gamma\left(r_{l}, r_{h}\right)$.

The log-characteristic function derived in (39) holds in general for a Poisson-Poisson clustered field of narrowband interferers, where the cluster centers are distributed over the parametric space $\Gamma\left(R_{l}, R_{h}\right)$ and the interferers are distributed over the parametric space $\Gamma\left(r_{l}, r_{h}\right)$ around each cluster center. The receiver location $R_{m}$ affects the inner expectation in (39). We now consider the same three cases, categorized by the region containing the cluster centers, and further simplify the $\log$-characteristic function. 
A. Case I: Cluster centers distributed over the entire plane $\left(R_{l}=0, R_{h} \rightarrow \infty\right)$

Consider a wireless network scenario, as shown in Fig. 2, where the center of interferer clusters are distributed according to a homogeneous spatial Poisson point process over the entire plane. Similar to Case I for a Poisson field of interferers, $\left\|R_{m}\right\|$ can be assumed to be zero without any loss in generality of the result. Conditioned on the number of clusters in $\Gamma\left(0, R_{h}\right)$, the distance of each cluster center from the origin follows the distribution

$$
f_{\mathbf{R}_{c} \mid \mathbf{K}_{c}}\left(R_{c} \mid K_{c}\right)= \begin{cases}\frac{2 R_{c}}{R_{h}^{2}} & \text { if } 0 \leq R_{c} \leq R_{h}, \\ 0 & \text { otherwise. }\end{cases}
$$

Thus as $R_{h} \rightarrow \infty$, with high probability, the distance of an interferer from the receiver can be approximated as $\mathbf{r}=\| \mathbf{R}_{c}+$ $\mathbf{R}_{c, f}\|\approx\| \mathbf{R}_{c} \|$. Expanding the expectation over $\mathbf{R}_{c}$ in (39), and using the Taylor series expansion of $e^{x}$, we have

$$
\begin{gathered}
\psi_{\overline{\mathbf{Y}}}(\bar{\omega}) \\
=\lim _{R_{h} \rightarrow \infty} A_{c}\left[\int_{0}^{R_{h}} e^{A_{f}\left(\mathbb{E}\left\{J_{0}\left(|\bar{\omega}| R_{c}^{-\frac{\gamma}{2}} \mathbf{h B}\right)\right\}-1\right)} \frac{2 R_{c}}{R_{h}^{2}} d R_{c}-1\right] \\
=e^{-A_{f}} \sum_{k=0}^{\infty} \frac{A_{f}^{k}}{k !}\left[\operatorname { l i m } _ { R _ { h } \rightarrow \infty } A _ { c } \left(\int_{0}^{R_{h}}\left(\mathbb{E}\left\{J_{0}\left(|\bar{\omega}| R_{c}^{-\frac{\gamma}{2}} \mathbf{h B}\right)\right\}\right)^{k}\right.\right. \\
\left.\left.\times \frac{2 R_{c}}{R_{h}^{2}} d R_{c}-1\right)\right] \\
=e^{-A_{f}} \sum_{k=0}^{\infty} \frac{A_{f}^{k}}{k !}
\end{gathered}
$$

where

$\Upsilon=\lim _{R_{h} \rightarrow \infty} A_{c}\left(\int_{0}^{R_{h}}\left(\mathbb{E}\left\{J_{0}\left(|\bar{\omega}| R_{c}^{-\frac{\gamma}{2}} \mathbf{h B}\right)\right\}\right)^{k} \frac{2 R_{c}}{R_{h}^{2}} d R_{c}-1\right)$.

Integrating the above by parts, reordering terms, and noting that $\lim _{R_{h} \rightarrow \infty} A_{c}\left[\left(\mathbb{E}\left\{J_{0}\left(|\bar{\omega}| R_{h}^{-\frac{\gamma}{2}} \mathbf{h B}\right)\right\}\right)^{k}-1\right]=0$ for $\gamma>$ 2 , we have

$\Upsilon=\lim _{R_{h} \rightarrow \infty}-\lambda_{c} \pi \int_{0}^{R_{h}} \frac{\partial}{\partial R_{c}}\left[\left(\mathbb{E}\left\{J_{0}\left(|\bar{\omega}| R_{c}^{-\frac{\gamma}{2}} \mathbf{h B}\right)\right\}\right)^{k}\right] R_{c}^{2} d R_{c}$.

Invoking the identity (17), and approximating $\boldsymbol{\Lambda}(|\bar{\omega}|))<<1$ for $|\bar{\omega}|$ close to zero, we note that

$$
\begin{aligned}
{\left[\mathbb{E}\left\{J_{0}\left(|\bar{\omega}| R_{c}^{-\frac{\gamma}{2}} \mathbf{h B}\right)\right\}\right]^{k} } & =e^{-\frac{|\bar{\omega}|^{2} k R_{c}^{-\gamma} \mathbb{E}\left\{\mathbf{h}^{2} \mathbf{B}^{2}\right\}}{4}}(1+\mathbf{\Lambda}(|\bar{\omega}|))^{k} \\
& \approx \mathbb{E}\left\{J_{0}\left(|\bar{\omega}| \sqrt{k} R_{c}^{-\frac{\gamma}{2}} \mathbf{h B}\right)\right\} .
\end{aligned}
$$

Substituting (44) in (42), and noting that $\frac{d}{d x} J_{0}(x)=-J_{1}(x)$, we get

$$
\Upsilon=-|\bar{\omega}|^{\frac{4}{\gamma}} \lambda_{c} \pi(\sqrt{k})^{\frac{4}{\gamma}} \mathbb{E}_{\mathbf{h}, \mathbf{B}}\left\{\mathbf{h}^{\frac{4}{\gamma}} \mathbf{B}^{\frac{4}{\gamma}}\right\} \int_{0}^{\infty} \frac{J_{1}(x)}{x^{\frac{4}{\gamma}}} d x .
$$

Using (45), the log-characteristic function in (40) reduces to

$$
\begin{array}{r}
\psi_{\overline{\mathbf{Y}}}(\bar{\omega})=-|\bar{\omega}|^{\frac{4}{\gamma}}\left[\left(\lambda_{c} \pi \mathbb{E}_{\mathbf{h}, \mathbf{B}}\left\{\mathbf{h}^{\frac{4}{\gamma}} \mathbf{B}^{\frac{4}{\gamma}}\right\} \int_{0}^{\infty} \frac{J_{1}(x)}{x^{\frac{4}{\gamma}}} d x\right) \times\right. \\
\left.\sum_{k=0}^{\infty} \frac{e^{-A_{f}} A_{f}^{k}(\sqrt{k})^{\frac{4}{\gamma}}}{k !}\right] .
\end{array}
$$

Equation (46) is the log-characteristic function of an isotropic symmetric alpha stable distribution centered at zero such that

$$
\psi_{\mathbf{Y}_{I}, \mathbf{Y}_{Q}}\left(\omega_{I}, \omega_{Q}\right)=-\sigma\left|\sqrt{\omega_{I}^{2}+\omega_{Q}^{2}}\right|^{\alpha}
$$

where $\alpha=\frac{4}{\gamma}$ is the characteristic exponent $(0<$ $\alpha<2)$, and $\sigma=\left[\left(\lambda_{c} \pi \mathbb{E}_{\mathbf{h}, \mathbf{B}}\left\{\mathbf{h}^{\alpha} \mathbf{B}^{\alpha}\right\} \int_{0}^{\infty} \frac{J_{1}(x)}{x^{\alpha}} d x\right)\right.$ $\left.\sum_{k=0}^{\infty} \frac{e^{-A_{f}} A_{f}^{k}(\sqrt{k})^{\alpha}}{k !}\right]$ is the dispersion parameter $(\sigma>0)$ of the symmetric alpha stable distribution [25]. Hence, when the center of interferer clusters are distributed according to a spatial Poisson process on the entire plane, the co-channel interference follows a symmetric alpha stable distribution. Note that unlike Case I for a Poisson field of interferers, the symmetric alpha stable distribution is not an exact model due to approximation in (44), but accurately models the tail probability of the interference.

\section{B. Case II: Cluster centers distributed over finite-area annular region $\left(0 \leq R_{l}<R_{h}<\infty\right.$, and $R_{m} \notin \Gamma\left(R_{l}-r_{h}, R_{h}+r_{h}\right)$ )}

Consider a wireless network scenario, as shown in Fig. 2, where the cluster centers are distributed over a finite-area annular region. The receiver location is such that it does not belong to the space of active interferers $\left(R_{m} \notin \Gamma\left(R_{l}-\right.\right.$ $\left.\left.r_{h}, R_{h}+r_{h}\right)\right)$. Similar to Case II for a Poisson field of interferers, this scenario is useful in characterizing interference from a finite-area annular field when the receiver is located interior to the region with a guard zone (when $\left\|R_{m}\right\|<R_{l}-r_{h}$ ) or at a point exterior to the region (when $\left\|R_{m}\right\|>R_{h}+r_{h}$ ).

Using the identity (17), the log-characteristic function in (39) can be expressed as

$$
\begin{array}{r}
\psi_{\overline{\mathbf{Y}}}(\bar{\omega})=A_{c}\left[\mathbb { E } _ { \mathbf { R } _ { c } } \left\{\operatorname { e x p } \left(A _ { f } \left(e^{\frac{-|\bar{\omega}|^{2} \mathbb{E}_{\mathbf{R}_{c, f}, \mathbf{h}, \mathbf{B}}\left\{\mathbf{r}^{-\gamma} \mathbf{h}^{2} \mathbf{B}^{2}\right\}}{4} \times}\right.\right.\right.\right. \\
(1+\boldsymbol{\Lambda}(|\bar{\omega}|))-1))\}-1]
\end{array}
$$

where $\boldsymbol{\Lambda}(|\bar{\omega}|)$ is the correction term given by (18). For notational simplicity, let $\mathbf{F}=\mathbb{E}_{\mathbf{R}_{c, f}, \mathbf{h}, \mathbf{B}}\left\{\mathbf{r}^{-\gamma} \mathbf{h}^{2} \mathbf{B}^{2}\right\} . \mathbf{F}$ is then a function of the random variable $\mathbf{R}_{c}$. Approximating $\boldsymbol{\Lambda}(|\bar{\omega}|))<<1$ for $|\bar{\omega}|$ in the neighborhood of zero, and using the Taylor series expansion of $e^{x}$, the log-characteristic function reduces to

$$
\begin{aligned}
\psi_{\overline{\mathbf{Y}}}(\bar{\omega}) & \approx A_{c}\left[\mathbb{E}_{\mathbf{R}_{c}}\left\{e^{-A_{f}} \sum_{k=0}^{\infty} \frac{A_{f}^{k}}{k !} e^{\frac{-k|\bar{\omega}|^{2} \mathbf{F}}{4}}\right\}-1\right] \\
& =A_{c}\left[e^{-A_{f}} \sum_{l=0}^{\infty} \frac{(-1)^{l}|\bar{\omega}|^{2 l} \mathbb{E}_{\mathbf{R}_{c}}\left\{\mathbf{F}^{l}\right\}}{4^{l} l !} \sum_{k=0}^{\infty} \frac{A_{f}^{k} k^{l}}{k !}-1\right] .
\end{aligned}
$$


To express the log-characteristic function in closed form, we approximate $\mathbb{E}_{\mathbf{R}_{c}}\left\{\mathbf{F}^{l}\right\} \approx\left(\mathbb{E}_{\mathbf{R}_{c}}\{\mathbf{F}\}\right)^{l}$. This approximation holds with equality for $l=0,1$ and hence does not affect the coefficient of $|\bar{\omega}|^{2}$ term. The coefficient of the lowest order term affected by this approximation is the $|\bar{\omega}|^{4}$ term. Thus the log-characteristic function is not severely affected by this approximation for $|\bar{\omega}|$ in the neighborhood of zero, which is desired for accurately modeling the tail probability, and can be expressed as

$$
\psi_{\overline{\mathbf{Y}}}(\bar{\omega}) \approx A_{c}\left[\exp \left(A_{f}\left(e^{\frac{-|\bar{\omega}|^{2} \mathbb{E}_{\mathbf{R}_{c}}\{\mathbf{F}\}}{4}}-1\right)\right)-1\right] .
$$

Using the log-characteristic function, and using the Taylor series expansion from $e^{x}$, the characteristic function can be expressed as

$$
\Phi_{\overline{\mathbf{Y}}}(\bar{\omega})=e^{-A_{c}} \sum_{l=0}^{\infty} \frac{A_{f}^{l}}{l !}\left(\sum_{k=0}^{\infty} \frac{A_{c}^{k} k^{l} e^{-k A_{f}}}{k !}\right) e^{\frac{-l|\bar{\omega}|^{2} \mathbb{E}_{\mathbf{R}_{c}}\{\mathbf{F}\}}{4}} .
$$

Equation (52) is the characteristic function of an isotropic Gaussian mixture model such that

$$
\Phi_{\mathbf{Y}_{I}, \mathbf{Y}_{Q}}\left(\omega_{I}, \omega_{Q}\right)=\sum_{l=0}^{\infty} p_{l} e^{\frac{-\left(\omega_{I}^{2}+\omega_{Q}^{2}\right) \sigma_{l}^{2}}{2}}
$$

where $p_{l}=\frac{e^{-A_{c}} A_{f}^{l}}{l !}\left(\sum_{k=0}^{\infty} \frac{A_{c}^{k} k^{l} e^{-k A_{f}}}{k !}\right)$ are the mixture prob-

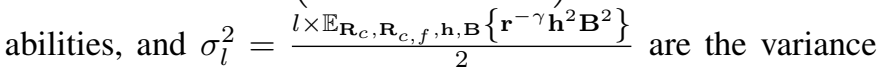
of the individual Gaussian components, for $l \geq 0$.

The two approximations involved in expressing the true loglikelihood function (48) as (51) are approximating $\Lambda(|\bar{\omega}|)<<$ 1 for $|\bar{\omega}|$ in the neighborhood of zero, and expressing $\mathbb{E}_{\mathbf{R}_{c}}\left\{\mathbf{F}^{l}\right\}$ as $\left(\mathbb{E}_{\mathbf{R}_{c}}\{\mathbf{F}\}\right)^{l}$. Using (18), the coefficient of $|\bar{\omega}|^{4}$ term in the true log-characteristic function (48) can be expressed as

$$
A_{c} e^{-A_{f}}\left[\frac{\mathbb{E}_{\mathbf{R}_{c}}\left\{\mathbf{F}^{2}\right\}}{32} \sum_{k=0}^{\infty} \frac{k^{2} A_{f}^{k}}{k !}+\mathbb{E}_{\mathbf{R}_{c}}\left\{\mathbf{c}_{4}\right\} \sum_{k=0}^{\infty} \frac{k A_{f}^{k}}{k !}\right]
$$

where $\mathbf{c}_{4}=\frac{\mathbb{E}_{\mathbf{R}_{c, f}, \mathbf{h}, \mathbf{B}}\left\{\mathbf{r}^{-2 \gamma} \mathbf{h}^{4} \mathbf{B}^{4}\right\}-2\left(\mathbb{E}_{\mathbf{R}_{c, f}, \mathbf{h}, \mathbf{B}}\left\{\mathbf{r}^{-\gamma} \mathbf{h}^{2} \mathbf{B}^{2}\right\}\right)^{2}}{128}$, and $\mathbf{F}=\mathbb{E}_{\mathbf{R}_{c, f}, \mathbf{h}, \mathbf{B}}\left\{\mathbf{r}^{-\gamma} \mathbf{h}^{2} \mathbf{B}^{2}\right\}$. Comparing with the coefficient of the $|\bar{\omega}|^{4}$ term in the approximated log-characteristic function (51), the Gaussian mixture distribution provides a good approximation to the interference statistics in this scenario when

$$
\begin{aligned}
&\left|\frac{\operatorname{Var}(\mathbf{F})}{32} \sum_{k=0}^{\infty} \frac{k^{2} A_{f}^{k}}{k !}+\mathbb{E}_{\mathbf{R}_{c}}\left\{\mathbf{c}_{4}\right\} \sum_{k=0}^{\infty} \frac{k A_{f}^{k}}{k !}\right|<< \\
&\left|\frac{\left(\mathbb{E}_{\mathbf{R}_{c}}\{\mathbf{F}\}\right)^{2}}{32} \sum_{k=0}^{\infty} \frac{k^{2} A_{f}^{k}}{k !}\right|
\end{aligned}
$$

where $\operatorname{Var}(\mathbf{F})=\mathbb{E}_{\mathbf{R}_{c}}\left\{\mathbf{F}^{2}\right\}-\left(\mathbb{E}_{\mathbf{R}_{c}}\{\mathbf{F}\}\right)^{2}$. Intuitively, the above condition is satisfied when the interferers are not close to the receiver (i.e., $\left\|R_{m}\right\|<<R_{l}-r_{h}$ or $\left\|R_{m}\right\|>>R_{h}+r_{h}$ ) and $R_{h}$ is not very high compared to $R_{l}$ when $\left\|R_{m}\right\|<R_{l}-r_{h}$.
C. Case III: Cluster centers distributed over infinite-area annular region with guard zone $\left(R_{l}>0, R_{h} \rightarrow \infty\right.$, and $\left.\left\|R_{m}\right\|<R_{l}-r_{h}\right)$

Consider a wireless network, as shown in Fig. 2, where the center of interferer clusters are distributed according to a homogeneous spatial Poisson point process over the entire plane, except within a guard zone around the receiver. Analogous to Case III for a Poisson field of interferers, the distance of each cluster center from the origin follows the distribution

$$
f_{\mathbf{R}_{c} \mid \mathbf{K}_{c}}\left(R_{c} \mid K_{c}\right)= \begin{cases}\frac{2 R_{c}}{R_{h}^{2}-R_{l}^{2}} & \text { if } R_{l} \leq R_{c} \leq R_{h}, \\ 0 & \text { otherwise. }\end{cases}
$$

Thus as $R_{h} \rightarrow \infty$, with high probability, the distance of an interferer from receiver located at $R_{m}$ can be approximated as $\mathbf{r}=\left\|\mathbf{R}_{c}+\mathbf{R}_{c, f}-R_{m}\right\| \approx\left\|\mathbf{R}_{c}\right\|$, particularly for $R_{m}<<$ $R_{l}-r_{h}$. Analogous to Case I, on expanding the expectation over $\mathbf{R}_{c}$ in (39), and using the Taylor series expansion for $e^{x}$, we have

$$
\begin{aligned}
\psi_{\overline{\mathbf{Y}}}(\bar{\omega}) & =\lim _{R_{h} \rightarrow \infty} A_{c}\left[\int_{R_{l}}^{R_{h}} e^{A_{f}\left(\mathbb{E}\left\{J_{0}\left(|\bar{\omega}| R_{c}^{-\frac{\gamma}{2}} \mathbf{h B}\right)\right\}-1\right)} \times\right. \\
\left.\frac{2 R_{c}}{R_{h}^{2}-R_{l}^{2}} d R_{c}-1\right] & \\
& =e^{-A_{f}} \sum_{k=0}^{\infty} \frac{A_{f}^{k}}{k !} \Upsilon
\end{aligned}
$$

where

$$
\begin{array}{r}
\Upsilon=\lim _{R_{h} \rightarrow \infty} A_{c}\left(\int_{R_{l}}^{R_{h}}\left(\mathbb{E}\left\{J_{0}\left(|\bar{\omega}| R_{c}^{-\frac{\gamma}{2}} \mathbf{h B}\right)\right\}\right)^{k} \times\right. \\
\left.\frac{2 R_{c}}{R_{h}^{2}-R_{l}^{2}} d R_{c}-1\right) .
\end{array}
$$

Integrating the above by parts, reordering terms, and noting that $\lim _{R_{h} \rightarrow \infty} \lambda_{c} \pi R_{h}^{2}\left[\left(\mathbb{E}\left\{J_{0}\left(|\bar{\omega}| R_{h}^{-\frac{\gamma}{2}} \mathbf{h B}\right)\right\}\right)^{k}-1\right]=0$ for $\gamma>$ 2 , we have

$$
\begin{aligned}
& \Upsilon=-\lambda_{c} \pi R_{l}^{2}\left(\left(\mathbb{E}_{\mathbf{h}, \mathbf{B}}\left\{J_{0}\left(|\bar{\omega}| R_{l}^{-\frac{\gamma}{2}} \mathbf{h B}\right)\right\}\right)^{k}-1\right)- \\
& \lim _{R_{h} \rightarrow \infty} \lambda_{c} \pi \int_{R_{l}}^{R_{h}} \frac{\partial}{\partial R_{c}}\left[\left(\mathbb{E}_{\mathbf{h}, \mathbf{B}}\left\{J_{0}\left(|\bar{\omega}| R_{c}^{-\frac{\gamma}{2}} \mathbf{h B}\right)\right\}\right)^{k}\right] R_{c}^{2} d R_{c} .
\end{aligned}
$$

Invoking the identity (17), approximating $\Lambda(|\bar{\omega}|)<<1$ for $|\bar{\omega}|$ in the neighborhood of zero, and using the Taylor series expansion of $e^{x}$, we have

$$
\begin{array}{r}
\Upsilon \approx \lambda_{c} \pi R_{l}^{2}\left[\sum_{m=1}^{\infty} \frac{(-1)^{m}|\bar{\omega}|^{2 m} k^{m}}{4^{m} m !}\left(\mathbb{E}\left\{\mathbf{h}^{2} \mathbf{B}^{2}\right\}\right)^{m} \times\right. \\
\left.R_{l}^{-\gamma m} \frac{2}{\gamma m-2}\right] .
\end{array}
$$

Similar to Case III for Poisson field of interferers, the $\frac{2}{\gamma m-2}$ multiplicative factor inside the summation prevents $\Upsilon$, and 
hence the log-characteristic function, to be expressed in closed form. We thus approximate the function $\frac{2}{\gamma m-2}$ as $\eta e^{\beta m}$ for $m \geq 1$, where $\{\eta, \beta\}$ are chosen to minimize a weighted mean squared error (WMSE) criterion as discussed in Section III-C. Using this approximation, (59) reduces to

$$
\Upsilon \approx \lambda_{c} \pi R_{l}^{2} \eta\left(e^{\frac{-l|\bar{\omega}|^{2} R_{l}^{-\gamma} e^{\beta} \mathbb{E}\left\{\mathbf{h}^{2} \mathbf{B}^{2}\right\}}{4}}-1\right)
$$

Substituting the above equation in (56), the log-characteristic function can be expressed as

$\psi_{\overline{\mathbf{Y}}}(\bar{\omega})=\lambda_{c} \pi R_{l}^{2} \eta\left[\exp \left(A_{f}\left(e^{\frac{-|\bar{\omega}|^{2} R_{l}^{-\gamma} e^{\beta} \mathbb{E}\left\{\mathbf{h}^{2} \mathbf{B}^{2}\right\}}{4}}-1\right)\right)-1\right]$.

Using the log-characteristic function, and the Taylor series expansion for $e^{x}$, the characteristic function can be expressed as

$$
\begin{array}{r}
\Phi_{\overline{\mathbf{Y}}}(\bar{\omega})=e^{-\lambda_{c} \pi R_{l}^{2} \eta} \sum_{l=0}^{\infty}\left[\frac{A_{f}^{l}}{l !}\left(\sum_{k=0}^{\infty} \frac{\left(\lambda_{c} \pi R_{l}^{2} \eta\right)^{k} k^{l} e^{-k A_{f}}}{k !}\right) \times\right. \\
\left.e^{\frac{-l|\bar{\omega}|^{2} R_{l}^{-\gamma} e^{\beta} \mathbb{E}\left\{\mathbf{h}^{2} \mathbf{B}^{2}\right\}}{4}}\right] . \quad \text { (62) }
\end{array}
$$

Equation (62) is the characteristic function of an isotropic Gaussian mixture model such that

$$
\Phi_{\mathbf{Y}_{I}, \mathbf{Y}_{Q}}\left(\omega_{I}, \omega_{Q}\right)=\sum_{l=0}^{\infty} p_{l} e^{\frac{-\left(\omega_{I}^{2}+\omega_{Q}^{2}\right) \sigma_{l}^{2}}{2}}
$$

where $p_{l}=\frac{e^{-\lambda_{c} \pi R_{l}^{2} \eta} A_{f}^{l}}{l !}\left(\sum_{k=0}^{\infty} \frac{\left(\lambda_{c} \pi R_{l}^{2} \eta\right)^{k} k^{l} e^{-k A_{f}}}{k !}\right)$ are the mixture probabilities, and $\sigma_{l}^{2}=\frac{l \times R_{l}^{-\gamma} e^{\beta} \mathbb{E}_{\mathbf{h}, \mathbf{B}}\left\{\mathbf{h}^{2} \mathbf{B}^{2}\right\}}{2}$ are the variance of the individual Gaussian components, for $l \geq 0$.

Using (58), (17), and (18), the coefficient of $|\bar{\omega}|^{4}$ term in the true log-characteristic function (56) can be expressed as

$$
\begin{array}{r}
\lambda_{c} \pi R_{l}^{-2 \gamma+2} e^{-A_{f}}\left[\frac{(\mathbb{E}\{\mathbf{Z}\})^{2}}{32} \sum_{k=0}^{\infty} \frac{k^{2} A_{f}^{k}}{k !}+c_{4} \sum_{k=0}^{\infty} \frac{k A_{f}^{k}}{k !}\right] \times \\
\left(\frac{2}{2 \gamma-2}\right) \cdot(64)
\end{array}
$$

where $\mathbf{Z}=\mathbf{h}^{2} \mathbf{B}^{2}$ and $c_{4}=\frac{\mathbb{E}\left\{\mathbf{Z}^{2}\right\}-2(\mathbb{E}\{\mathbf{Z}\})^{2}}{128}$. Comparing with the coefficient of $|\bar{\omega}|^{4}$ term in the approximated logcharacteristic function (62), the Gaussian mixture distribution provides a good approximation to the interference statistics in this scenario when

$$
\begin{aligned}
& \mid \frac{(\mathbb{E}\{\mathbf{Z}\})^{2}}{32}\left(\frac{2}{2 \gamma-2}-\eta e^{2 \beta}\right) \sum_{k=0}^{\infty} \frac{k^{2} A_{f}^{k}}{k !}+ \\
& \frac{2 c_{4}}{(2 \gamma-2)} \sum_{k=0}^{\infty} \frac{k A_{f}^{k}}{k !}|<<| \frac{(\mathbb{E}\{\mathbf{Z}\})^{2}}{32} \eta e^{2 \beta} \sum_{k=0}^{\infty} \frac{k^{2} A_{f}^{k}}{k !} \mid .
\end{aligned}
$$

Analogous to Case III for a Poisson field of interferers, the above condition is independent of the parameter $R_{l}$ that governs the interference space and is satisfied when the variance of the random variable $\mathbf{h}^{2} \mathbf{B}^{2}$ is low when compared to $\left[\mathbb{E}\left\{\mathbf{h}^{2} \mathbf{B}^{2}\right\}\right]^{2}$. Note that the above condition does not capture the error due to the approximation $\mathbf{r}=\left\|\mathbf{R}_{c}+\mathbf{R}_{c, f}-R_{m}\right\| \approx$
$\mathbf{R}_{c}$, which is true with high probability and is particularly valid for $\left\|R_{m}\right\|<<R_{l}-r_{h}$.

\section{Summary AND DiscuSSION}

Tables III and IV summarize the key results derived in this paper for a field of Poisson and Poisson-Poisson cluster distributed interferers, respectively. We now make the following observations.

1. Narrowband emissions from interferers: The narrowband form of the interfering emissions is truly attributed to the narrowband filtering done at the receiver. Hence the interferer emissions can have a higher bandwidth than the receiver, as long as the transients caused due to interferer emissions at the receiver can be ignored [19]. From [19], the analysis and results presented in this paper are valid as long as the duration of the interfering emissions $\left(T_{I}\right)$ is much greater than the reciprocal of the receiver bandwidth $\left(\Delta f_{R}\right)$, i.e., $T_{I}>>\frac{1}{\Delta f_{R}}$.

2. Extensions for finite-area interference fields with arbitrary shape: The finite-area cases are studied for Poisson and Poisson-Poisson clustered field of interferers in Sections III-B and IV-B, respectively. For a finite-area interference $\Gamma$ with arbitrary shape, $\mathbb{P}\{k$ in $\Gamma\}=\lambda|\Gamma|$, where $|\Gamma|$ denotes the area of the space $\Gamma$ in (7) and (34). The remaining analysis does not change since we do not expand the expectation over the random variable $\mathbf{r}$ for finite-area cases. Hence it can be readily shown that Middleton Class A and the Gaussian mixture models are still applicable for interference spaces with arbitrary shape using the following changes in the parameters. The overlap index for Middleton Class $\mathrm{A}$ is expressed more generally as $A=\lambda|\Gamma|$ for finite-area field of Poisson distributed interferers. For finite-area field of PoissonPoisson cluster distributed interferers, the parameters $A_{f}=\lambda_{f}\left|\Gamma_{f}\right|$ and $A_{c}=\lambda_{c}\left|\Gamma_{c}\right|$, where $\Gamma_{c}$ is the space in which the cluster centers are distributed and $\Gamma_{f}$ is the space in which the interferers are distributed around each cluster center.

\section{Simulation Results}

Using the physical model discussed in Section II, we apply Monte-Carlo numerical techniques to simulate the co-channel interference observed at the receiver in various wireless network environments based on (1). At each sample instant, the location of the active interferers is generated as a realization of a spatial Poisson or Poisson-Poisson cluster point process. Parameter values governing the interference space and the receiver location change according to the wireless network model under consideration. It should be noted that parameters denoting distance are are treated as dimensionless quantities as this does not influence the statistics of the resultant interference.

System model parameters used in the numerical simulations are

$\gamma=4, \mathbf{h} \sim \mathcal{C N}(0,1), \lambda=10^{-4}, \lambda_{c}=10^{-4}, \lambda_{f}=10^{-3}$ 
TABLE III: Statistical-physical modeling of co-channel interference in a field of Poisson distributed interferers categorized by the region containing the interferers.

\begin{tabular}{|c|c|c|c|}
\hline \multicolumn{4}{|c|}{ Poisson field of Interferers } \\
\hline Wireless Scenario & Example Wireless Network & Statistical Model & Statistics Modeled \\
\hline $\begin{array}{l}\text { Case I: Entire Plane } \\
\left(r_{l}=0, r_{h} \rightarrow \infty\right)\end{array}$ & Sensor or Ad hoc networks & $\begin{array}{l}\text { Symmetric Alpha Stable } \\
\text { Parameters: } \\
\alpha=\frac{4}{\gamma} \\
\sigma=\lambda \pi \mathbb{E}_{\mathbf{h}, \mathbf{B}}\left\{\mathbf{h}^{\alpha} \mathbf{B}^{\alpha}\right\} \int_{0}^{\infty} \frac{J_{1}(x)}{x^{\alpha}} d x\end{array}$ & exact statistics \\
\hline $\begin{array}{l}\text { Case II: Finite-area Annular } \\
\text { Region } \\
\left(0 \leq r_{l}<r_{h}<\infty \text {, and }\right. \\
\left.R_{m} \notin \Gamma\left(r_{l}, r_{h}\right)\right)\end{array}$ & $\begin{array}{l}\text { a. Cellular networks (out-of-cell } \\
\text { interference) } \\
\text { b. Interference from a hotspot } \\
\text { (e.g. cafe) }\end{array}$ & $\begin{array}{l}\text { Middleton Class A } \\
\text { Parameters: } \\
A=\lambda \pi\left(r_{h}^{2}-r_{l}^{2}\right) \\
\Omega_{2 A}=\frac{A \times \mathbb{E}_{\mathbf{r}, \mathbf{h}, \mathbf{B}}\left\{\mathbf{r}^{-\gamma} \mathbf{h}^{2} \cdot \mathbf{B}^{2}\right\}}{2} \\
\text { where } \mathbf{r}=\left\|\mathbf{R}-R_{m}\right\|\end{array}$ & $\begin{array}{l}\text { tail probability } \\
\text { when (24) is met }\end{array}$ \\
\hline $\begin{array}{l}\text { Case III: Infinite-area with } \\
\text { Guard Zone } \\
\left(r_{l}>0, r_{h} \rightarrow \infty \text {, and }\right. \\
\left.\left\|R_{m}\right\|<r_{l}\right)\end{array}$ & $\begin{array}{l}\text { a. Cellular networks (out-of-cell } \\
\text { interference) } \\
\text { b. Decentralized networks with } \\
\text { contention-based MAC protocols } \\
\text { c. Dense WiFi networks }\end{array}$ & $\begin{array}{l}\text { Middleton Class A } \\
\text { Parameters: } \\
A=\lambda \pi r_{l}^{2} \eta \\
\Omega_{2 A}=\frac{A \times r_{l}^{-\gamma} e^{\beta} \mathbb{E}_{\mathbf{h}, \mathbf{B}}\left\{\mathbf{h}^{2} \cdot \mathbf{B}^{2}\right\}}{2} \\
\text { where }\{\eta, \beta\} \text { are obtained from (29). }\end{array}$ & $\begin{array}{l}\text { tail probability } \\
\text { when (32) is met }\end{array}$ \\
\hline
\end{tabular}

TABLE IV: Statistical-physical modeling of co-channel interference in a field of Poisson-Poisson cluster distributed interferers categorized by the region containing the cluster centers.

\begin{tabular}{|c|c|c|c|}
\hline \multicolumn{4}{|c|}{$\begin{array}{c}\text { Poisson-Poisson Cluster field of Interferers } \\
\end{array}$} \\
\hline Wireless Scenario & Example Wireless Network & Statistical Model & Statistics Modeled \\
\hline $\begin{array}{l}\text { Case I: Entire Plane } \\
\left(R_{l}=0, R_{h} \rightarrow \infty\right)\end{array}$ & $\begin{array}{l}\text { a. Two-tier femtocell networks } \\
\text { (femtocell interference) } \\
\text { b. Sensor or ad hoc networks with } \\
\text { geographical or MAC induced } \\
\text { clustering }\end{array}$ & 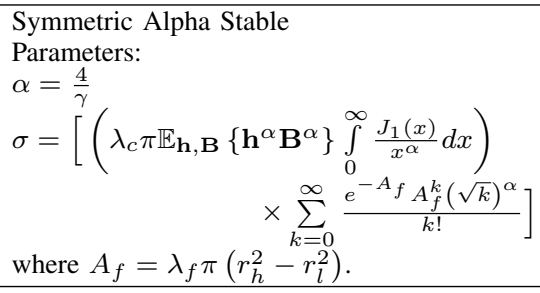 & tail probability \\
\hline $\begin{array}{l}\text { Case II: Finite-area Annular } \\
\text { Region } \\
\left(0 \leq R_{l}<R_{h}<\infty \text {, and }\right. \\
\left.R_{m} \notin \Gamma\left(R_{l}-r_{h}, R_{h}+r_{h}\right)\right)\end{array}$ & $\begin{array}{l}\text { a. Cellular networks (out-of-cell } \\
\text { interference) with user clustering } \\
\text { b. Interference from region with } \\
\text { multiple (random) hotspots } \\
\text { (e.g. market place, university) }\end{array}$ & 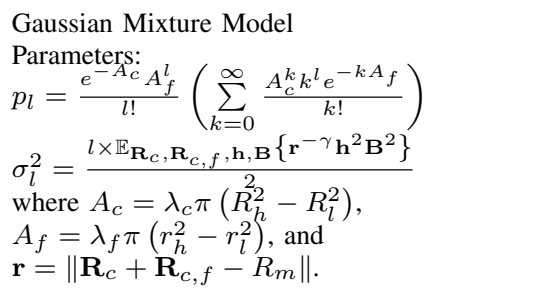 & $\begin{array}{l}\text { tail probability } \\
\text { when (54) is met }\end{array}$ \\
\hline $\begin{array}{l}\text { Case III: Infinite-area with } \\
\text { Guard Zone } \\
\left(R_{l}>0, R_{h} \rightarrow \infty \text {, and }\right. \\
\left.\left\|R_{m}\right\|<R_{l}-r_{h}\right)\end{array}$ & $\begin{array}{l}\text { a. Two-tier femtocell networks } \\
\text { (out-of-cell femtocell interference) } \\
\text { b. Cellular networks (out-of-cell } \\
\text { interference) with user clustering }\end{array}$ & $\begin{array}{l}\text { Gaussian Mixture Model } \\
\text { Parameters: } \\
p_{l}=\frac{e^{-\lambda_{c} \pi R_{l}^{2} \eta} A_{f}^{l}}{l !} \sum_{k=0}^{\infty} \frac{\left(\lambda_{c} \pi R_{l}^{2} \eta\right)^{k} k^{l} e^{-k A_{f}}}{k !} \\
\sigma_{l}^{2}=\frac{l \times R_{l}^{-\gamma} e^{\beta} \mathbb{E}_{\mathbf{h}, \mathbf{B}}\left\{\mathbf{h}^{2} \mathbf{B}^{2}\right\}}{2} \\
\text { where } A_{f}=\lambda_{f} \pi\left(r_{h}^{2}-r_{l}^{2}\right),\{\eta, \beta\} \text { are } \\
\text { obtained from (29). }\end{array}$ & $\begin{array}{l}\text { tail probability } \\
\text { when }(65) \text { is met }\end{array}$ \\
\hline
\end{tabular}

The amplitude of the interferer emissions, B, was chosen as a constant for a particular wireless environment such that the tail probability, $\mathbb{P}(\|\mathbf{Y}\|>y)$, at an interference threshold of $y=7$, is of the order of $10^{-4}$. The probability distribution of co-channel interference is empirically estimated from 500000 time samples of the received interference using kernel smoothed density estimators [33].

Accuracy of the statistical models is established by comparing the empirical and interference model tail probabilities. We compare the asymptotic decay rates of the tail probabilities given by

$$
\rho(y)=-\frac{\log (\mathbb{P}(\|\mathbf{Y}\|>y))}{y}
$$

where $\rho(y)$ is the asymptotic decay rate at interference amplitude $y$. The decay rate is the rate at which the tail probability asymptotically approaches zero. The decay rates are a useful measure to compare the extreme value statistics of different statistical models with respect to the empirically estimated distribution.

Accuracy of fit of the statistical models is also quantified using the Kulback-Liebler divergence (KLD) measure [34], where a KLD of zero indicates an exact match of the densities. 


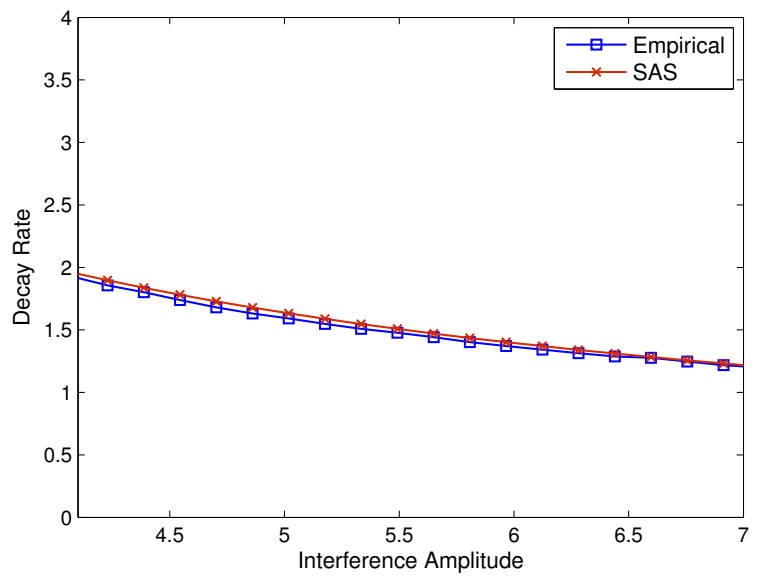

Fig. 3: Decay rates for tail probabilities of simulated co-channel interference and the symmetric alpha stable (SAS) model for Case $I\left(r_{l}=0, r_{h}=\right.$ $\infty, \mathbf{B}=5$ ) of Poisson field of interferers. The Middleton Class A and Gaussian models are not suitable in this scenario as the mean intensity $\Omega_{2 A} \rightarrow \infty$.

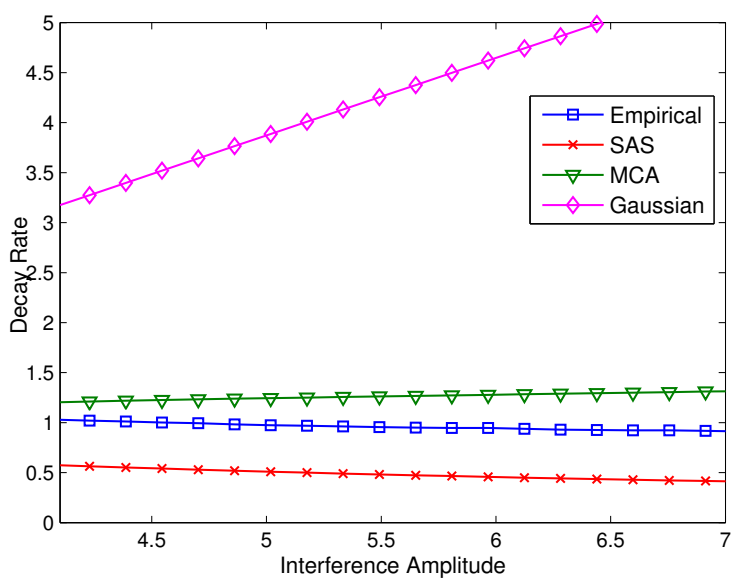

Fig. 4: Decay rates for tail probabilities of simulated co-channel interference and the symmetric alpha stable (SAS), Middleton Class A (MCA), and Gaussian models for Case II $\left(r_{l}=20, r_{h}=40,\left\|R_{m}\right\|=4, \mathbf{B}=1400\right)$ of Poisson field of interferers. MCA has the best match to the empirical (simulated) co-channel interference.

Lower KLD, however, does not imply correspondence in tail probabilities since the KLD is the relative error between two distribution functions over their entire support. Thus, even though a statistical model has a low KLD with respect to the empirical distribution, it may be an inaccurate model for modeling extreme statistics.

\section{A. Co-channel interference in a Poisson field of interferers}

Figs. 3, 4, and 5 show the decay rates of the empirical distribution compared with the statistical models for Case I, Case II, and Case III (see Fig. 1), respectively. In each scenario, we compare the empirical distribution against the symmetric alpha stable and the Middleton Class A distribution with appropriate parameters (see Table III), and a Gaussian distribution with equal variance.

For a Poisson field of interferers, the results demonstrate that the tail probabilities of the co-channel interference in Case I

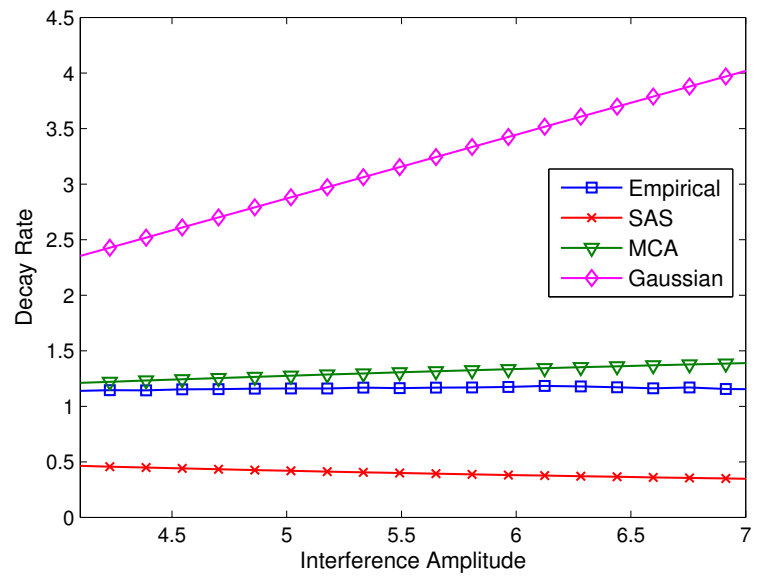

Fig. 5: Decay rates for tail probabilities of simulated co-channel interference and the symmetric alpha stable (SAS), Middleton Class A (MCA), and Gaussian models for Case III $\left(r_{l}=30, r_{h}=\infty,\left\|R_{m}\right\|=4, \mathbf{B}=2200\right)$ of Poisson field of interferers. $\{\eta, \beta\}=\{2.781,-1.025\}$ for $\gamma=4$ and $u(k)=e^{-k}$ from Table II. MCA has the best match to the empirical (simulated) co-channel interference.

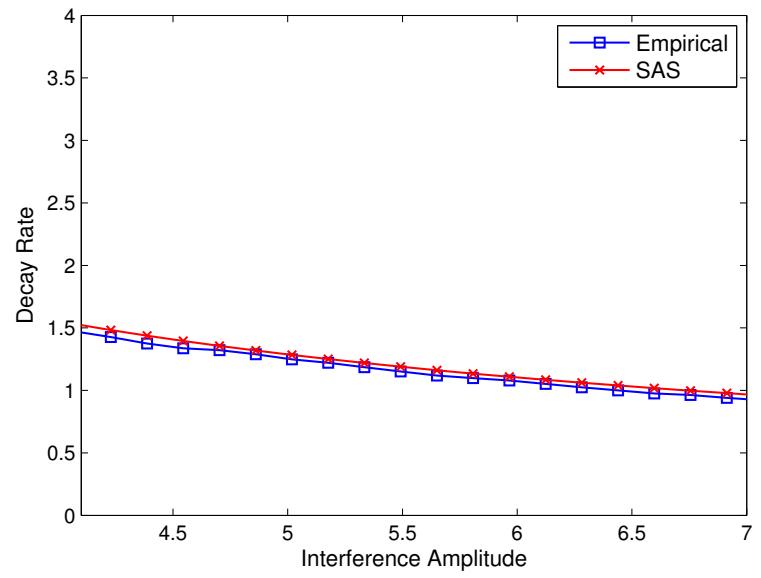

Fig. 6: Decay rates for tail probabilities of simulated co-channel interference and the symmetric alpha stable (SAS) model for Case $I\left(R_{l}=0, R_{h}=\right.$ $\left.\infty, r_{l}=0, r_{h}=10, \mathbf{B}=100\right)$ of Poisson-Poisson cluster field of interferers. The Gaussian mixture and Gaussian models are not suitable in this scenario as the mean intensity $\Omega_{2 A} \rightarrow \infty$.

are well modeled using a symmetric alpha distribution, while the Middleton Class A distribution provides a good fit to the tail probabilities in Case II and Case III.

\section{B. Co-channel interference in a Poisson-Poisson cluster field of interferers}

Figs. 6, 7, and 8 show the decay rates of the empirical distribution compared with the statistical models for Case I, Case II, and Case III (see Fig. 2), respectively. In each scenario, we compare the empirical distribution against the symmetric alpha stable and the Gaussian mixture distribution with appropriate parameters (see Table IV). Further, we compare the empirical distribution of co-channel interference to a Gaussian distribution with equal variance for all scenarios.

For a Poisson-Poisson clustered field of interferers, the results demonstrate that the tail probabilities of the co-channel 


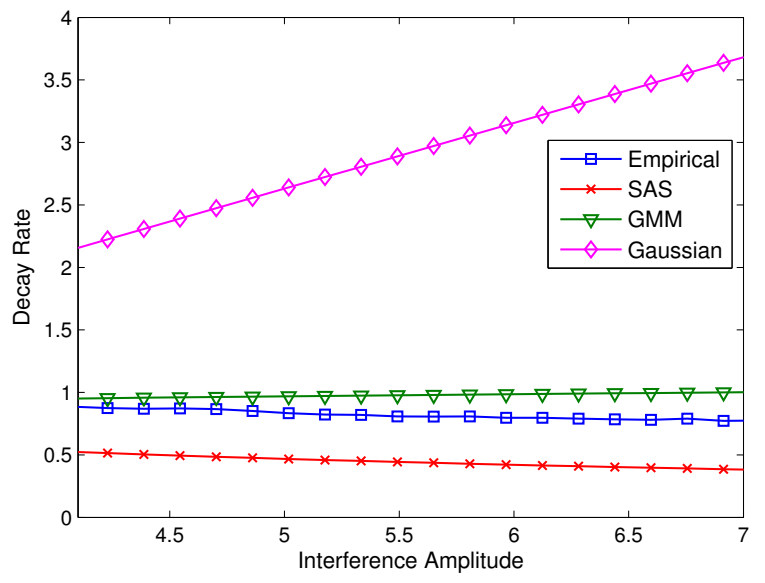

Fig. 7: Decay rates for tail probabilities of simulated co-channel interference and the symmetric alpha stable (SAS), Gaussian mixture (GMM), and Gaussian models for Case II $\left(R_{l}=40, R_{h}=80, r_{l}=0, r_{h}=10,\left\|R_{m}\right\|=\right.$ $4, \mathbf{B}=6000)$ of Poisson-Poisson cluster field of interferers. GMM has the best match to the empirical (simulated) co-channel interference.

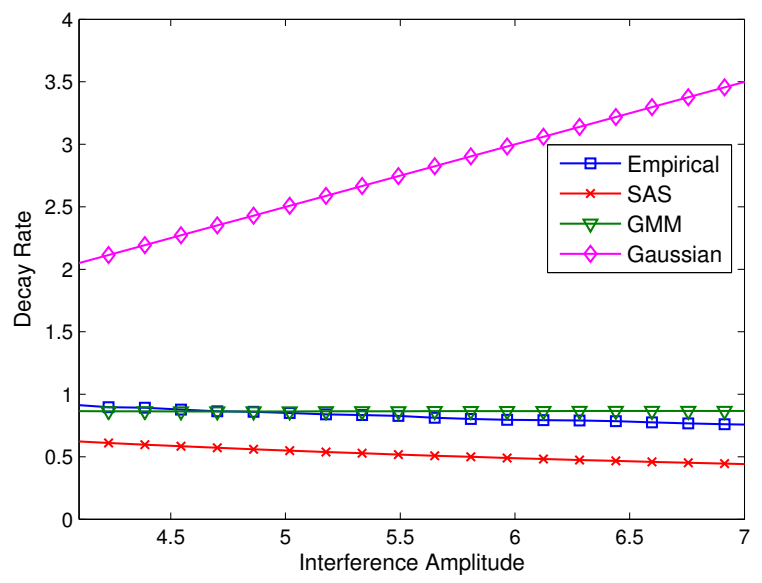

Fig. 8: Decay rates for tail probabilities of simulated co-channel interference and the symmetric alpha stable (SAS), Gaussian mixture (GMM), and Gaussian models for Case III $\left(R_{l}=30, R_{h} \rightarrow \infty, r_{l}=0, r_{h}=\right.$ $\left.10,\left\|R_{m}\right\|=4, \mathbf{B}=4000\right)$ of Poisson-Poisson cluster field of interferers. $\{\eta, \beta\}=\{2.781,-1.025\}$ for $\gamma=4$ and $u(k)=e^{-k}$ from Table II. MCA has the best match to the empirical (simulated) co-channel interference.

interference in Case I are well modeled using a symmetric alpha distribution, while the Gaussian mixture distribution provides a good fit to the tail probabilities in Case II and Case III.

\section{Comments on simulation results}

In all of the network models discussed above, the statistics of co-channel interference are not modeled well by the Gaussian distribution. The Gaussian distribution decays far too quickly to accurately model the impulsive nature of co-channel interference.

For Case II of Poisson and Poisson-Poisson cluster distributed interferers, accuracy of the Middleton Class A and the Gaussian mixture models in approximating the tail probability of co-channel interference depends on the interference space based on (24) and (54), respectively. The results shown in
TABLE V: Kulback-Liebler divergence between empirical and statistical model distribution (joint in-phase and quadrature-phase distribution) in Poisson and Poisson-Poisson cluster field of interferers for different wireless network scenarios. Here SAS, MCA, and GMM stand for symmetric alpha stable, Middleton Class A, and Gaussian mixture model, respectively. Parameter values governing the interference space for each of the scenarios are listed in caption to Figs. 3 through 8 .

\begin{tabular}{|c|c|c|c|}
\hline \multicolumn{5}{|c|}{ Poisson Field of Interferers } \\
\hline Wireless Scenario & SAS & MCA & Gaussian \\
\hline \hline Case I & 0.0154 & - & - \\
\hline Case II & 0.0953 & 0.0141 & 0.2275 \\
\hline Case III & 0.1594 & 0.8869 & 0.2246 \\
\hline Poisson-Poisson Cluster Field of Interferers \\
\hline Wireless Scenario & SAS & GMM & Gaussian \\
\hline \hline Case I & 0.1656 & - & - \\
\hline Case II & 0.1243 & 0.0182 & 0.2789 \\
\hline Case III & 0.3309 & 3.2177 & 0.6234 \\
\hline
\end{tabular}

Figs. 4 and 7 are when these conditions are met with moderate accuracy. For example, the Middleton Class A and the Gaussian mixture models provides a much closer approximation to the simulated tail probabilities for $\left\|R_{m}\right\|=0$, with the remaining parameters held constant.

For Case III, even though the Middleton Class A and the Gaussian mixture models closely approximate the tail probability of the simulated interference (see Figs. 5 and 8), Table V shows that the KL-divergence form the empirical distribution is significantly higher than the other statistical models. This is because the approximations used for accurately modeling the tail probabilities may introduce significant mismatch in approximated distribution for near-zero amplitudes (discrete probability mass of $e^{-A}$ and $e^{-A_{c}\left(1-e^{-A_{f}}\right)}$ at zero amplitude in this case for Poisson and Poisson-Poisson clustered interferers, respectively).

\section{CONCLUSION}

The results presented in this paper are applicable to a wide variety of wireless network topologies, including user clustering, contention-based and contention-free MAC protocols, and finite-area interference regions. Tables III and IV lists some of the example wireless networks for which the results are applicable. Knowledge of closed form amplitude statistics of co-channel interference can be used to analyze and improve the communication performance of wireless networks, including both physical (PHY) layer algorithms and medium access control (MAC) layer protocols.

To elaborate on the applications of the results, consider the example of a wireless ad hoc network with contentionbased MAC protocol that creates a guard zone around the receiver [15]. This corresponds to Case III in Fig. 1, in which the Middleton Class A distribution was shown to accurately model tail probability of the interference. Increasing the guard zone size decreases the interference to the desired transmitterreceiver pair, thereby improving the communication performance for that pair. The density of such transmitter-receiver pairs, however, is reduced due to increased guard zones in the network. Thus an optimum guard zone size exists for which the network throughput, defined as the product of the density of active transmitter-receiver pairs and the probability of success of a typical pair, is maximized.

Regarding the analysis and design of MAC protocol, the 
closed form Middleton Class A distribution derived for Case III can be used to derive closed form expressions for communication performance measures such as outage probability. Further, the optimum guard zone size that maximizes the network throughput for a desired communication performance (e.g. upper bound on outages) can also be derived analytically. The authors in [15] demonstrated $2-100 \times$ improvement in network throughput by using optimal guard zone size over non-contention based MAC protocols such as ALOHA [6].

PHY layer methods to improve the communication performance include designing receiver filtering and detection rules to mitigate the interference, by treating interference as noise. For example, the authors in [4] derived the BER optimal Bayesian detection rule in the presence of Middleton Class A noise and demonstrated $10-100 \times$ reduction in BER. This increases the network throughput by increasing the probability of success for any given density of users. Receiver filtering and detection methods are generally designed using the knowledge of the form of interference distribution only, and ignore dependence of the distribution parameters on the system model parameters (such as density of users). This is because many of the system model parameters, such as user density, may not be directly observable. The parameters of the interference distribution can be estimated using parameter estimation algorithm at the receiver on received interference samples collected by listening to the environment.

\section{APPENDIX A}

StATISTICAL PROPERTIES OF THE SYMMETRIC ALPHA Stable, Gaussian Mixture, and Middleton Class A MODELS

\section{A. Symmetric Alpha Stable Model}

A complex random variable $\mathbf{Y}=\mathbf{Y}_{I}+j \mathbf{Y}_{Q}$ is said to follow an isotropic symmetric alpha stable distribution centered at zero if the joint characteristic function of its inphase and quadrature-phase components can be expressed as [25]

$$
\Phi_{\mathbf{Y}_{I}, \mathbf{Y}_{Q}}\left(\omega_{I}, \omega_{Q}\right)=e^{-\sigma\left|\sqrt{\omega_{I}^{2}+\omega_{Q}^{2}}\right|^{\alpha}}
$$

where $\alpha$ is the characteristic function with $0<\alpha \leq 2$, and $\sigma$ $(\sigma>0)$ is the dispersion parameter.

Closed form expressions for the probability distribution function, however, do not exist except for the cases $\alpha=2$ (Gaussian distribution) and $\alpha=1$ (Cauchy distribution).

An isotropic symmetric alpha stable random variable $\mathbf{Y}$ can be expressed in a sub-Gaussian form as $\mathbf{Y}=\mathbf{A}^{\frac{1}{2}}\left(\mathbf{G}_{1}+j \mathbf{G}_{2}\right)$, where $\mathbf{G}_{1}$ and $\mathbf{G}_{2}$ are i.i.d. zero-mean univariate Gaussian random variables and $\mathbf{A}$ is a positive stable random variable with characteristic exponent $\frac{\alpha}{2}$ and dispersion $\cos ^{2}\left(\frac{\pi \alpha}{4}\right)$ [25]. The tail probability of the envelope random variable $\|\mathbf{Y}\|$ can then be expressed as

$\mathbb{P}_{S \alpha S}(\|\mathbf{Y}\|>y)=y^{-\alpha} \sigma\left(2 \cos \left(\frac{\pi \alpha}{4}\right)\right)^{\alpha} C\left(\frac{\alpha}{2}\right) \Gamma\left(1+\frac{\alpha}{2}\right)$

as $y \rightarrow \infty$, where

$$
C(\alpha)= \begin{cases}\frac{2}{\pi} & \text { when } \alpha=1, \\ \frac{1-\alpha}{\Gamma(2-\alpha) \cos \left(\frac{\pi \alpha}{2}\right)} & \text { otherwise. }\end{cases}
$$

\section{B. Gaussian Mixture Model}

The joint probability density function of a complex random variable $\mathbf{Y}=\mathbf{Y}_{I}+j \mathbf{Y}_{Q}$ centered at zero and distributed according to an isotropic Gaussian mixture model can be expressed as

$$
f_{\mathbf{Y}_{I}, \mathbf{Y}_{Q}}\left(y_{I}, y_{Q}\right)=p_{0} \delta\left(y_{I}\right) \delta\left(y_{Q}\right)+\sum_{l=1}^{\infty} p_{l} \frac{1}{\sigma_{l} \sqrt{2 \pi}} e^{-\frac{y_{I}^{2}+y_{Q}^{2}}{2 \sigma_{l}^{2}}}
$$

where $p_{l}$ are the mixture probabilities such that $p_{l} \geq 0$ and $\sum_{l=0}^{\infty} p_{l}=1, \sigma_{l}^{2}$ is the variance of the individual Gaussian components of the mixture density, and $\delta(\cdot)$ represents the Dirac delta functional.

From (69), the two dimensional characteristic function can be expressed as

$$
\Phi_{\mathbf{Y}_{I}, \mathbf{Y}_{Q}}\left(\omega_{I}, \omega_{Q}\right)=p_{0}+\sum_{l=1}^{\infty} p_{l} e^{\frac{-\left(\omega_{I}^{2}+\omega_{Q}^{2}\right) \sigma_{l}^{2}}{2}}
$$

Using (69), the tail probability of the random envelope for the Gaussian mixture distribution with parameters $p_{l}$ and $\sigma_{l}^{2}$ $\left(\sigma_{l}^{2} \geq 0\right)$ for $y \geq 0$ can be expressed as

$$
\mathbb{P}_{G M M}(\|\mathbf{Y}\|>y)=\sum_{l=1}^{\infty} p_{l} e^{-\frac{y^{2}}{2 \sigma_{l}^{2}}}
$$

\section{Middleton Class A Model}

The Middleton Class A distribution is a particular form of the Gaussian mixture distribution. The joint probability density function of a isotropic complex random variable $\mathbf{Y}=\mathbf{Y}_{I}+j \mathbf{Y}_{Q}$ distributed according to Middleton Class A model (without an additive Gaussian component) can be expressed as [19]

$$
f_{\mathbf{Y}_{I}, \mathbf{Y}_{Q}}\left(y_{I}, y_{Q}\right)=e^{-A} \delta\left(y_{I}\right) \delta\left(y_{Q}\right)+\sum_{m=1}^{\infty} \frac{e^{-A} A^{m}}{m !} e^{-\frac{y_{I}^{2}+y_{Q}^{2}}{2 m \Omega_{2 A}}}
$$

where $A$ is the overlap index and $\Omega_{2 A}$ is the mean intensity of the random variable.

From (72), the joint characteristic function of the in-phase and quadrature phase components of the complex random variable can be expressed as

$$
\Phi_{\mathbf{Y}_{I}, \mathbf{Y}_{Q}}\left(\omega_{I}, \omega_{Q}\right)=e^{A\left(e^{-\frac{\left(\omega_{I}^{2}+\omega_{Q}^{2}\right) \Omega_{2 A}}{2 A}}-1\right)} .
$$

Note that as $A \rightarrow \infty$ while $\Omega_{2 A}$ is finite, the Middleton Class A model converges to a Gaussian distribution with variance $\Omega_{2 A}$.

Using (72), the tail probability for the Middleton Class A distribution with parameters $A$ and $\Omega_{2 A}$ corresponding to an amplitude threshold $y \geq 0$ can be expressed as

$$
\mathbb{P}_{M C A}(\|\mathbf{Y}\|>y)=\sum_{m=1}^{\infty} \frac{e^{-A} A^{m}}{m !} e^{-\frac{y^{2}}{2 m \Omega_{2 A}}}
$$




\section{REFERENCES}

[1] S. Weber, J. G. Andrews, and N. Jindal, "The effect of fading, channel inversion, and threshold scheduling on ad hoc networks," IEEE Transactions on Information Theory, vol. 53, no. 11, pp. 4127-4149, Nov. 2007.

[2] K. Gulati, A. Chopra, R. W. Heath, B. L. Evans, K. R. Tinsley, and $X$. E. Lin, "MIMO receiver design in the presence of radio frequency interference," in Proc. IEEE Global Communications Conference, Nov. 30-Dec. 42008.

[3] C. L. Nikias and M. Shao, Signal Processing with Alpha-Stable Distributions and Applications. John Wiley \& Sons, 1995.

[4] A. Spaulding and D. Middleton, "Optimum reception in an impulsive interference enviroment-part I: Coherent detection," IEEE Transactions on Communications, vol. 25, no. 9, pp. 910-923, 1977.

[5] R. S. Blum, R. J. Kozick, and B. Sadler, "An adaptive spatial diversity receiver for non-Gaussian interference and noise," IEEE Transactions on Signal Processing, vol. 47, no. 8, pp. 2100-2111, Aug. 1999.

[6] F. Baccelli and B. Błaszczyszyn, "Stochastic geometry and wireless networks, volume 2 - applications," in Foundations and Trends in Networking. Now Publishers Inc., 2009, vol. 4, no. 1-2, pp. 1-312.

[7] K. Gulati, M. Nassar, A. Chopra, B. Okafor, M. DeYoung, N. Aghasadeghi, A. Sujeeth, and B. L. Evans, "Radio frequency interference modeling and mitigation toolbox in MATLAB," Version 1.4, Feb. 2010. [Online]. Available: http://users.ece.utexas.edu/ bevans/ projects/rfi/software/

[8] F. Baccelli, M. Klein, M. Lebourges, and S. Zuyev, "Stochastic geometry and architecture of communication networks," Journal of Telecommunication Systems, vol. 7, pp. 209-227, Jun. 1997.

[9] M. Haenggi and R. K. Ganti, "Interference in large wireless networks," in Foundations and Trends in Networking. Now Publishers Inc., 2008, vol. 3, no. 2, pp. 127-248.

[10] M. Z. Win, P. C. Pinto, and L. A. Shepp, "A mathematical theory of network interference and its applications," Proceedings of the IEEE, vol. 97, no. 2, pp. 205-230, Feb. 2009.

[11] F. Baccelli and B. Błaszczyszyn, "Stochastic geometry and wireless networks, volume 1 - theory," in Foundations and Trends in Networking. Now Publishers Inc., 2009, vol. 3, no. 3-4, pp. 249-449.

[12] V. Chandrasekhar, J. G. Andrews, and A. Gatherer, "Femtocell networks: a survey," IEEE Communications Magazine, vol. 46, no. 9, pp. 59-67, Sep. 2008.

[13] V. Chandrasekhar and J. G. Andrews, "Uplink capacity and interference avoidance for two-tier femtocell networks," IEEE Transactions on Wireless Communications, vol. 8, no. 7, pp. 3498-3509, Jul. 2009.

[14] X. Yang and G. de Veciana, "Inducing multiscale spatial clustering using multistage MAC contention in spread spectrum ad hoc networks," IEEE/ACM Transactions on Networking, vol. 15, no. 6, pp. 1387-1400, Dec. 2007.

[15] A. Hasan and J. G. Andrews, "The guard zone in wireless ad hoc networks," IEEE Transactions on Wireless Communications, vol. 4 no. 3, pp. 897-906, Mar. 2007.

[16] IEEE ComSoc LAN MAN Standards Committee, Wireless LAN Medium Access Control (MAC) and Physical Layer (PHY) Specifications: IEEE Standard 802.11, The Institute of Electrical and Electronics Engineers, 1997.

[17] E. Salbaroli and A. Zanella, "Interference analysis in a Poisson field of nodes of finite area," IEEE Transactions on Vehicular Technology, vol. 58, no. 4, pp. 1776-1783, May 2009.

[18] D. Middleton, "Statistical-physical models of electromagnetic interference," U.S. Department of Commerce, Office of Telecommunications, Tech. Rep., Apr. 1976.

[19] — - "Non-Gaussian noise models in signal processing for telecommunications: New methods and results for class A and class B noise models," IEEE Transactions on Information Theory, vol. 45, no. 4, pp. 1129-1149, May 1999.

[20] E. S. Sousa, "Performance of a spread spectrum packet radio network link in a Poisson field of interferers," IEEE Transactions on Information Theory, vol. 38, no. 6, pp. 1743-1754, Nov. 1992.

[21] J. Ilow and D. Hatzinakos, "Analytic alpha-stable noise modeling in a Poisson field of interferers or scatterers," IEEE Transactions on Signal Processing, vol. 46, no. 6, pp. 1601-1611, Jun. 1998.

[22] X. Yang and A. Petropulu, "Co-channel interference modeling and analysis in a Poisson field of interferers in wireless communications," IEEE Transactions on Signal Processing, vol. 51, no. 1, pp. 64-76, Jan. 2003.
[23] K. Gulati, A. Chopra, B. L. Evans, and K. R. Tinsley, "Statistical modeling of co-channel interference," in Proc. IEEE Global Communications Conference, Nov. 30-Dec. 42009.

[24] K. Gulati, B. L. Evans, and K. R. Tinsley, "Statistical modeling of co-channel interference in a field of Poisson distributed interferers," in Proc. IEEE International Conference on Acoustics, Speech, and Signal Processing, Mar. 14-19 2010.

[25] G. Samorodnitsky and M. S. Taqqu, Stable Non-Gaussian Random Processes: Stochastic Models with Infinite Variance. Chapman and Hall, New York, 1994.

[26] R. Ganti and M. Haenggi, "Interference and outage in clustered wireless ad hoc networks," IEEE Transactions on Information Theory, vol. 55, no. 9, pp. 4067-4086, Sep. 2009.

[27] S. O. Rice, "Mathematical analysis of random noise," Bell Systems Technical Journal, vol. 23, pp. 282-332, 1944.

[28] M. Westcott, "On the existence of a generalized shot-noise process," in Studies in Probability and Statistics: Papers in Honour of Edwin J. G. Pitman, Amsterdam, 1976, pp. 73-88.

[29] S. B. Lowen and M. C. Teich, "Power-law shot noise," IEEE Transactions on Information Theory, vol. 36, no. 6, pp. 1302-1318, Nov. 1990.

[30] J. Venkataraman, M. Haenggi, and O. Collins, "Shot noise models for outage and throughput analysis in wireless ad hoc networks," in Proc. Military Communications Conference, Oct. 2006, pp. 1-7.

[31] R. Baldick, Applied Optimization: Formulation and Algorithms for Engineering Systems. Cambridge University Press, 2006.

[32] D. J. Daley and D. Vere-Jones, An Introduction to the Theory of Point Processes, 1st ed. Springer, New York, 1988.

[33] Z. I. Botev, "A novel nonparametric density estimator," The University of Queensland, Australia, Tech. Rep., Nov. 2006.

[34] T. M. Cover and J. A. Thomas, Elements of Information Theory, 2nd ed. Wiley \& Sons, New York, 2006.

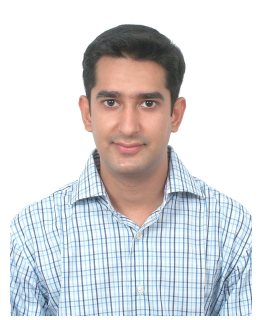

Kapil Gulati (S'06) received the B.Tech. degree in Electronics and Communications Engineering from the Indian Institute of Technology, Guwahati in 2004 and the M.S. degree in Electrical Engineering from the University of Texas at Austin in 2008. $\mathrm{He}$ is currently pursuing the Ph.D. degree at The University of Texas at Austin. From 2004 to 2006, he was employed as a Hardware Design Engineer at Texas Instruments, India.

Since 2006, he has been a Research Assistant at the Embedded Signal Processing Laboratory at The University of Texas at Austin. His current research interests include signal processing for communication systems in the presence of non-Gaussian impulsive noise and MIMO communications.

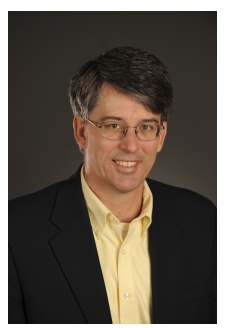

Brian L. Evans (S'87, M'93, SM'97, F'09) is the Engineering Foundation Professor in the Department of Electrical and Computer Engineering (ECE) at The University of Texas at Austin. He received the BS double major in Electrical Engineering and Computer Science from the Rose-Hulman Institute of Technology in 1987, and the M.S. and Ph.D. degrees in Electrical Engineering from the Georgia Institute of Technology in 1988 and 1993, respectively. $\mathrm{He}$ was a post-doctoral researcher at the University of California, Berkeley, 1993-1996, and joined the faculty at UT Austin in 1996.

Prof. Evans received the National Science Foundation CAREER award in 1997 and the ECE Gordon Lepley IV Memorial Teaching Award at UT Austin in 2008. He has graduated $16 \mathrm{PhD}$ and $8 \mathrm{MS}$ students. He has published more than 190 refereed conference and journal papers. His current research interests include radio frequency interference mitigation for wireless systems; real-time multichannel multicarrier testbeds for ADSL and powerline communication systems; image display algorithms for handheld reflective displays; and electronic design automation tools for multicore embedded systems. 


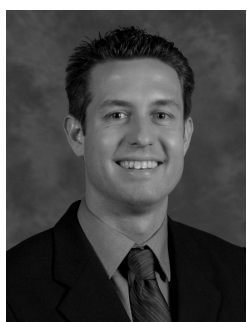

Jeffrey Andrews (S'98, M'02, SM'06) received the B.S. in Engineering with High Distinction from Harvey Mudd College in 1995, and the M.S. and Ph.D. in Electrical Engineering from Stanford University in 1999 and 2002, respectively. He is an Associate Professor in the Department of Electrical and Computer Engineering at the University of Texas at Austin, and the Director of the Wireless Networking and Communications Group (WNCG), a research center comprising 17 faculty and 10 industrial affiliates. He developed Code Division Multiple Access systems at Qualcomm from 1995-97, and has consulted for entities including the WiMAX Forum, Microsoft, Apple, Clearwire, Palm, ADC, and NASA.

Dr. Andrews is co-author of two books, Fundamentals of WiMAX (PrenticeHall, 2007) and Fundamentals of LTE (Prentice-Hall, 2010), and holds the Earl and Margaret Brasfield Endowed Fellowship in Engineering at UT Austin, where he received the ECE departments first annual High Gain award for excellence in research. He is a Senior Member of the IEEE, and served as an associate editor for the IEEE Transactions on Wireless Communications from 2004-08.

Dr. Andrews received the National Science Foundation CAREER award in 2007 and is the Principal Investigator of a 9 university team of 12 faculty in DARPA's Information Theory for Mobile Ad Hoc Networks program. $\mathrm{He}$ has been co-author of four best paper award recipients, two at IEEE Globecom (2006 and 2009) one at Asilomar (2008), and the 2010 IEEE Communications Society Best Tutorial Paper Award. His research interests are in communication theory, information theory, and stochastic geometry applied to wireless ad hoc, femtocell and cellular networks.

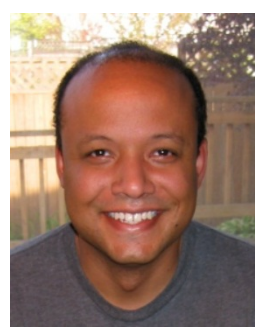

Kieth Tinsley (M’06, SM'09) is a Staff Engineer with Intel's Corporate Technology Group. Keith brings to Intel years of experience in design, development and research of communication systems ranging from satellites to experimental radios. His current work includes pioneering the development of adaptive algorithms to mitigate the impact of radio frequency interference (RFI) on wireless platforms. His current interests are in the areas of platform noise identification and in the development of estimation algorithms for wireless platform RFI immunity. He received his BSEE from Old Dominion University and his MSEE from Arizona State University. He is a member of Tau Beta Pi, Eta Kappa Nu, and IEEE. 